\title{
Modeling and Analysis of Wireless Power Transfer in Heterogeneous Cellular Networks
}

\author{
Yansha Deng, Member, IEEE, Lifeng Wang, Member, IEEE, Maged Elkashlan, Member, IEEE, \\ Marco Di Renzo, Senior Member, IEEE, and Jinhong Yuan, Fellow, IEEE
}

\begin{abstract}
In this paper, we model and analyze the downlink (DL) wireless power transfer and uplink (UL) information transmission of $K$-tier heterogeneous cellular networks (HCNs) with randomly located base stations (BSs) and mobile terminals (MTs). In the DL and UL, each energy-constrained MT pairs up with its corresponding BS, which provides the maximum received power at MT. Due to the densely located BSs and universal frequency reuse between all tiers in HCNs, the typical MT is allowed to harvest energy from the serving BS by direct beamforming, as well as from the other interfering BSs. Equipped with large storage battery, the typical MT utilizes the harvested energy to provide constant transmit power for the UL information transmission. Stochastic geometry is used to model and evaluate the intrinsic relationship between the energy harvested from the BSs in the DL and the information transmission performance in the UL. To well evaluate the system performance, we first derive exact expressions for the maximum transmit power at MT, the UL outage probability, and the UL average ergodic rate per MT. As the number of BS antennas goes to infinity, we further derive asymptotic expressions for the maximum transmit power at MT, the UL outage probability, and the UL average ergodic rate per MT. Our results show that the UL outage probability per MT first decreases and then increases with increasing the time allocation factor (the fraction of time allocated to the DL), and the UL outage probability and the UL average ergodic rate per MT can be largely improved by using the massive antenna arrays at the BSs.
\end{abstract}

Index terms- Heterogeneous cellular networks, radio frequency wireless power transfer, cell association, stochastic geometry, point process theory.

\section{INTRODUCTION}

The upsurge growth of smart phones, netbooks, tablets, and machine-to-machine (M2M) communication devices along with the increasing popularity of cloud and Web 2.0 multimedia infotainment applications (e.g., Google, Youtube,

Manuscript received May. 06, 2015; revised Jan. 05, 2016; accepted Jun. 21, 2016. This paper was presented in part at the Proc. IEEE Int. Conf. Commun. (ICC), Malaysia, Kuala Lumpur, Jun. 2016 [1]. This work was supported in part by the Australia Research Council (ARC) Discovery Project DP160104566. The editor coordinating the review of this manuscript and approving it for publication was Prof. Dawy Zaher.

Y. Deng and L. Wang were with the School of Electronic Engineering and Computer Science, Queen Mary University of London, London, UK (e-mail: yansha.deng@kcl.ac.uk; lifeng.wang@ucl.ac.uk).

M. Elkashlan is with the School of Electronic Engineering and Computer Science, Queen Mary University of London, London, UK (e-mail: maged.elkashlan@qmul.ac.uk).

Marco Di Renzo is with the Laboratoire des Signaux et Systèmes, CNRS, CentraleSupélec, Univ Paris Sud, Université Paris-Saclay, 3 rue Joliot Curie, Plateau du Moulon, 91192, Gif-sur-Yvette, France. (e-mail: marco.direnzo@12s.centralesupelec.fr).

J. Yuan is with School of Electrical Engineering and Telecommunications, University of New South Wales, Sydney, Australia (email: j.yuan@unsw.edu.au)
Facebook) lead to the rapid escalated energy consumption. This high depletion of energy evokes the rise of various energy harvesting technologies, which improve the energy efficiency of wireless networks and impose less detrimental effects on the environment at the same time. Traditional energy harvesting sources, such as solar, wind, hydroelectric, and thermoelectric may not always be accessible in some locations or environments. Alternatively, radio frequency (RF) wireless power transfer can be a cost-effective energy harvesting technique to prolong the lifetime of mobile devices and provide continuous and stable energy for wireless energy constrained networks [25]. This is done through harvesting energy from the ambient RF signals radiated by the transmitters $[6,7]$.

To achieve higher network capacity and increased spatial spectrum efficiency, traditional cellular systems are moving towards heterogeneous cellular networks (HCNs) [8]. In HCNs, several classes of BSs, including microcell base stations (BSs), picocell BSs, and femtocell BSs, are distributed throughout the conventional macrocell network to achieve seamless transmission between mobile devices, but this comes at the expense of increased interference [9-11]. The advent of HCNs marks a new era of viability for RF wireless power transfer in multi-tier cellular networks.

\section{A. Motivation}

In the coming $5 \mathrm{G}$, the densification of multi-tier $\mathrm{HCNs}$ will reduce the relative distance between BS and MT, which has the potential to fulfill the short range power transfer requirement reported in [2]. In downlink (DL) HCNs, each MT is associated with the BS which provides the maximum received power. As such, the serving BS acts as a dedicated RF energy source, similar to "power beacon" in [12]. Meanwhile, due to the universal frequency reuse, the typical MT also endures high levels of interference from the nearby interfering BSs. These densely deployed interfering BSs are typically located close to the typical MT, acting as ambient RF energy source for the MT [13]. The growing interests and breakthrough in deploying the large scale antenna in cellular networks with small cells enables the sharp beamforming and the low propagation loss, which in turn relieve the transmit power requirement of MT [2]. Inspired by the aforementioned reasons, RF wireless power transfer with direct beamforming can be a low-cost solution for sustainable operations of HCNs without modifying the hardware at the BS side.

\section{B. Related Works}

1) Single-cell WPCNs: One practical application of RF wireless power transfer is the wireless powered communica- 
tion network (WPCN), in which MTs perform uplink (UL) information transmission using the energy harvested from the DL wireless power transmission [14]. In [14], the harvest-thentransmit protocol was proposed where the distributed users with no other energy sources harvest energy from the hybrid access point (H-AP) with constant energy supply in the DL, and then utilize the stored energy to power the operating circuits and transmit information to the H-AP through time division multiple access (TDMA) in the UL. The work in [14] has been extended to WPCNs in [15] where all the users transmit to a multi-antenna H-AP via space-divisionmultiple-access (SDMA) using the energy harvested from the DL energy beamfoming. Various techniques such as user cooperation [16], full-duplex transmission [17], and massive MIMO [18] were proposed to enhance the performance of WPCNs.

2) Multi-cell WPCNs: Different from the aforementioned single-cell WPCNs [14-18], multi-cell WPCNs were studied in $[7,12,19,20]$. In [12], the energy sources, namely power beacons, were implemented in the existing cellular network to power the UL information transmission of mobile devices. In [19], a cognitive underlay device-to-device (D2D) communication in multi-channel cellular networks was proposed, where the D2D transmitters harvest RF energy from the DL and the UL transmissions from both the macrocell BSs and the cellular users. In [7], the mobile user with energy storage capability was powered by the ambient RF signals for UL transmission to the nearest single-antenna BS in HCNs, where the mobile user has high probability of experiencing power outage due to the insufficient energy harvested from the ambient RF. In fact, it was shown in [2] that energy harvested from ambient RF signals can only power small sensors with sporadic activities, while powering the mobile users needs to rely on direct beamforming.

3) Modeling of multi-tier HCNs: A new modeling approach based on stochastic geometry is now widely applied to capture the topological randomness of multi-tier HCNs, and it leads to tractable and simple analytical results [21]. In [22], it was shown that the DL coverage probability for the Poisson point process (PPP) model provides a lower bound for the counterpart with actual $4 \mathrm{G}$ deployment. To date, most of the literature have focused on the modeling of DL HCNs, considering fixed transmit power and fixed location of BSs in the DL transmission. Only [23,24] focused on the UL transmission of $K$-tier HCNs. In [23], a truncated channel inversion power control was applied at the user to ensure the same received power at the serving BSs. In [24], a novel cell association was proposed, which jointly maximizes the DL capacity and minimizes the MT power consumption.

\section{Contributions and Organization}

Inspired by the joint design of DL power transfer and UL information transmission in WPCNs in $[14,15]$, we focus on multi-cell wireless powered HCNs in which the multipleantenna BSs with direct beamforming provide RF energy to the single-antenna MTs without inherent energy supply in the DL. The MTs will then utilize the harvested energy to deliver information to the BSs with MRC in the UL. We summarize the main contributions of this paper as follows:

1) We formulate a tractable model for the DL wireless power transfer and the UL information transmission in HCNs. Assuming large storage battery in each MT, we derive an exact expression for the maximum transmit power of a typical MT associated with the $k$ th tier. We find that the maximum transmit power of typical MT that is associated with the $k$ th tier, is unaltered by increasing the number of antennas at the interfering BSs.

2) Based on the maximum transmit power, we derive exact expressions for the UL outage probability and the UL average ergodic rate per MT to characterize the link reliability and spectral efficiency. Our results show that the UL outage probability and the UL average ergodic rate per random MT may not necessarily improve with increasing the density of picocell BSs. It is interesting that the UL outage probability per MT first improves and then degrades with increasing the time allocation factor.

3) To examine the impact of massive antenna arrays at the BS, we derive the easy to compute expressions for the asymptotic UL averaged received power, asymptotic UL outage probability, and asymptotic UL average ergodic rate, as the number of antennas at the BSs goes to infinity. We confirm that the asymptotic and the exact results converge as the number of antennas grows large. The dramatic improvement of UL performance with the help of massive antenna arrays demonstrates the practical feasibility of wireless power transfer with direct beamforming in HCNs.

\section{SYSTEM DESCRIPTION}

We consider a $K$-tier $\mathrm{HCN}$ in which the BSs of each tier are spatially distributed in $\mathbb{R}^{2}$ according to an independent and homogenous Poisson point process (PPP), $\Phi_{k}$, with density $\lambda_{k}$. Each tier is assumed to have a different transmit power, $P_{t, k}$, a different path-loss exponent, $\eta_{k}$, and a different bias factor, $B_{k}$, for $k \in\{1, \cdots, K\}$, where $B_{k} \geq 1[25,26]$. Note that the transmit power value of BS, $P_{t, k}$, is for per channel use as assumed in $[19,23,27,28]$. In addition, the MTs are assumed to be spatially distributed in $\mathbb{R}^{2}$ according to a homogenous PPP, $\Phi_{U}^{\text {tot }}$ with density $\lambda_{u}^{\text {tot }}$. The density of MTs is assumed to be much higher that the overall density of BSs $\left(\lambda_{u}^{t o t} \gg \sum_{k=1}^{K} \lambda_{k}\right)$ in HCNs, and each MT has data ready for transmission, such that saturated traffic conditions hold [19,23,29]. It is further assumed that each BS is active and serves one active MT per channel, and the users within the same cell are scheduled based on time-division multiple-access (TDMA), such that the transmit power of BS is only devoted to serve one active MT. As remarked in [30], the dependence among the distances between the scheduled UEs and their serving BSs is weak and can be ignored, for mathematical tractability, it is assumed that the set of active MTs in each tier constitutes an independent homogeneous PPP as $\Phi_{U}$ [23]. Full-frequency reuse is also assumed to improve the spectrum efficiency [26]. 
The MTs and the BSs in the $k$ th tier are assumed to be equipped with 1 and $N_{k}$ antennas, respectively. Each MT has a rechargeable battery with large storage and is capable of converting the RF received power into a direct current (DC) signal to recharge the available battery. A harvest-thentransmit communication protocol for wireless power transfer is considered, where the MTs harvest energy from the BS in the DL, and then utilize the stored energy to power the operating circuits and transmit information to the BS [14]. More specifically, let $T$ denote the duration of a communication block, which consists of DL and UL transmissions. The first and second sub-blocks of duration $\alpha T$ and $(1-\alpha) T$ are allocated to DL and UL transmissions, respectively, where $\alpha$ $(0 \leq \alpha \leq 1)$ is the time allocation factor. In the DL, the typical MT harvests energy from all the active BSs of the $K$-tier HCNs. In the UL, the typical MT transmits information to its serving BS. The fading channels in DL and UL transmissions are assumed to be quasi-static, i.e., the fading channels are fixed within each sub-block and independently change from one block to another.

\section{A. Cell Association}

We aim to examine the performance of a random MT in HCNs. To facilitate the analysis, we usually consider a MT located at the origin, namely, typical MT. Without loss of generality, using the Slivnyak-Mecke's theorem [31], the biased-receivedpower (BRP), $P_{r, k}$, from a BS $x$ at the location $\mathbf{x} \in \mathbb{R}^{2}$ can be formulated as follows

$$
P_{r, k}=P_{t, k}\|\mathbf{x}\|^{-\eta_{k}} B_{k},
$$

where $\|\mathbf{x}\|$ denotes the BS-to-MT distance.

We consider a cell association criterion based on DL maximum BRP. According to this criterion, the BS offers the maximum received power for the typical MT is selected [25]. Accordingly, the selected serving BS for the typical MT $x^{*}$ can be formulated as follows

$$
\begin{gathered}
x^{*}=\underset{x \in\left\{x_{k}^{*}\right\}}{\arg \max } P_{t, k}\|\mathbf{x}\|^{-\eta_{k}} B_{k}, \\
\text { with } \quad x_{k}^{*}=\underset{x \in \Phi_{k}}{\arg \max } P_{t, k}\|\mathbf{x}\|^{-\eta_{k}}
\end{gathered}
$$

where $x_{k}^{*}$ for $k \in\{1, \ldots, K\}$ denotes the BS of tier $k$ that is closest to the MT, and $\Phi_{k}$ denotes the position sets of BSs in the $k$ th tier.

It is worth mentioning that: i) if $B_{k}=1$ for $k \in\{1, \ldots, K\}$, the MT is served by the BS providing the best received power, and ii) if $B_{k}=1 / P_{t, k}$, the MT is served by the BS providing the smallest path-loss [25].

\section{B. Downlink Power Transfer}

In the DL power transfer phase, each $N$-antenna BS uses maximal-ratio transmission (MRT) beamforming to transfer the power towards its own intended MT. The typical MT is located at the origin and denoted by $u_{0}$.

As stated in Remark 4.4 of [32], the widely used simplified attenuation function $\|\mathbf{x}\|^{-\eta_{j}}$ may result in infinite value at $\|\mathbf{x}\|=0$, and lost its accuracy for short distance and large intensity. Alternatively, we consider a short range propagation model for the calculation of received power at the intended MT to avoid the singularity caused by proximity between BSs and mobiles $[12,32]$. Note that this model also captures the realistic RF design constraints on the maximum received power, as widely assumed in 3GPP LTE Advanced standard $[33,34]$.

For a typical MT $\mathrm{u}_{0}$ located at the origin that is associated with its serving BS $S_{k_{x^{*}}}$ in the $k$ th tier, its received power is expressed as

$$
\begin{aligned}
& P_{\mathrm{r}_{\mathrm{u}_{0}, \mathrm{k}}}=\underbrace{P_{\mathrm{t}, S_{k_{x^{*}}}}\left\|\mathbf{h}_{S_{k_{x^{*}}}}\right\|^{2} L_{0}\left(\max \left\{\left\|\mathbf{x}_{S_{k_{x^{*}}}}\right\|, d\right\}\right)^{-\eta_{k}}}_{I_{S_{k_{x}}}}+ \\
& \underbrace{\sum_{j=1}^{K} \sum_{S_{x} \in \Phi_{j} \backslash S_{k_{x^{*}}}} P_{\mathrm{t}, S_{j}}\left|\mathbf{h}_{S_{x} u_{0}} \frac{\mathbf{g}_{S_{x} u_{j}}^{H}}{\left\|\mathbf{g}_{S_{x} u_{j}}\right\|}\right|^{2} L_{0}\left(\max \left\{\left\|\mathbf{x}_{S_{x} u_{0}}\right\|, d\right\}\right)^{-\eta_{j}}}_{I_{S_{x}}}
\end{aligned}
$$

where $d \geq 1$ is a constant value, $L_{0}$ is the path loss for a reference distance $r_{0}=1$, which is typically $(4 \pi / v)^{-2}$, and $v$ is the wavelength. In (3), $I_{S_{k_{x} *}}$ is the energy from the serving $\mathrm{BS}, I_{S_{x}}$ is the energy from the other BSs, $\mathbf{h}_{S_{k_{x^{*}}}} \in \mathcal{C}^{1 \times N}$ is the small-scale fading channel vector from the serving $\mathrm{BS}$ in the $k$ th tier to the typical MT, $\mathbf{h}_{S_{x} u_{0}} \in \mathcal{C}^{1 \times N}$ is the small-scale fading interfering channel vector from the other BS at a location $\mathbf{x}$ to the typical MT, and $\frac{\mathbf{g}_{S_{x} u_{j}}^{H}}{\left\|\mathbf{g}_{S_{x} u_{j}}\right\|}$ is the MRT beamforming vector of other BS at a location $\mathbf{x}$, where $\mathbf{g}_{S_{x} u_{j}} \in \mathcal{C}^{1 \times N}$ is the small-scale fading channel vector from the other BS at a location $\mathbf{x}$ to its associated MT. All the channels are assumed to experience Rayleigh fading such that $\left\|\mathbf{h}_{S_{k_{x} *}}\right\|^{2} \sim \operatorname{Gamma}(N, 1)$. According to [35], $\mathbf{h}_{S_{x} u_{0}} \frac{\mathbf{g}_{S_{x} u_{j}}^{H}}{\left\|\mathbf{g}_{S_{x} u_{j}}\right\|}$ is a zero-mean complex Gaussian variable, such that $\left|\mathbf{h}_{S_{x} u_{0}} \frac{\mathbf{g}_{S_{x} u_{j}}^{H}}{\left\|\mathbf{g}_{S_{x} u_{j}}\right\|}\right|^{2} \sim \exp (1)$.

\section{Uplink Information Transmission}

In the UL information transmission phase, the MTs remain associated with their serving BSs that powered them in the DL power transfer phase ${ }^{1}$, and consume the stored energy to transmit information signals to their serving BSs.

The large storage MTs are assumed to have very large battery capacity to enable reliable transmission power. As presented by Lemma 2 in [12] and the proof in [37], for MTs with large energy storage, the randomness of the instantaneous received power is averaged out and the active MTs in the $k$ th tier transmit signals continuously with fixed power $P_{\mathrm{t}_{\mathrm{u}}, k}$ up to $\phi \mathbb{E}\left\{P_{\mathrm{r}_{\mathrm{u}_{0}, \mathrm{k}}}\right\}$, where $\phi=\mu \frac{\alpha}{1-\alpha}{ }^{2}$. Here, we assume that the MT transmits signal with the maximum transmit power $\phi \mathbb{E}\left\{P_{\mathrm{r}_{\mathrm{u}_{0}, \mathrm{k}}}\right\}$, and the energy consumed for the signal transmission of MT should not exceed the harvested energy.

\footnotetext{
${ }^{1}$ In the current cellular networks, the UL cell association is based on DL cell association criterion [36].

${ }^{2}$ The processing power in the UL is ignored [14].
} 
Here, $\mu$ is the RF-to-DC conversion efficiency with $0<\mu<1$ [38], and all the MTs have the same $\mu$.

In the UL information transmission phase, each $N$-antenna BS employs maximal-ratio combiner (MRC) to combine the received signals. The signal-to-interference-plus-noise ratio (SINR) at the serving BS with MRC in the $k$ th tier is given by

$$
S I N R_{k}=\frac{\phi \mathbb{E}\left\{P_{r_{\mathrm{u}}, k}\right\}\left\|\mathbf{h}_{\mathrm{u}_{0}, S_{k_{x^{*}}}}\right\|^{2} L_{0}\left\|\mathbf{x}_{\mathrm{u}_{0}, S_{k_{x^{*}}}}\right\|^{-\eta_{k}}}{I_{U}+\delta^{2}},
$$

where $I_{U}=\sum_{j=1}^{K} I_{U, j}, I_{U, j}=\sum_{\mathrm{u}_{x, j} \in \tilde{\Phi}_{j} \backslash \mathrm{u}} \phi \mathbb{E}\left\{P_{r_{\mathrm{u}}, j}\right\}$

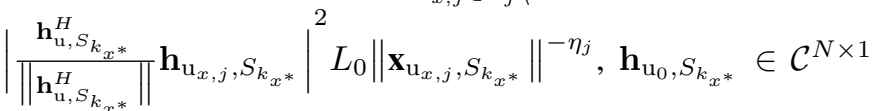
is the small-scale fading channel vector from the typical MT $\mathrm{u}_{0}$ to the serving BS in the $k$ th tier, $\mathbf{h}_{\mathrm{u}_{x, j}, S_{k_{r^{*}}}} \in \mathcal{C}^{N \times 1}$ is the small-scale fading channel vector from the interfering MT $\mathrm{u}_{x, j}$ in the $j$ th tier to the serving BS in the $k$ th tier, $\frac{\mathbf{h}_{\mathrm{u}_{0}, S_{k_{x^{*}}}^{H}}\left\|\mathbf{h}_{\mathrm{u}_{0}, S_{k_{x^{*}}}}\right\|}{\|}$ is the MRC vector of the serving BS in the $k$ th tier, $\tilde{\Phi}_{j}$ is the point process corresponding to the interfering MTs in the $j$ th tier, and $\delta^{2}$ is the noise power.

\section{System-Level Performance Evaluation of DOWNLINK POWER TRANSFER}

To determine the efficiency of DL wireless power transfer, we derive the maximum transmit power at the typical MT in the $k$ th tier based on (3) in the following theorem .

\section{A. Maximum Transmit Power}

Theorem 1. The maximum transmit power at the typical MT associated with the $k$ th tier BS using BRP cell association is given by

$$
\begin{aligned}
P_{\mathrm{t}_{\mathrm{u}}, k}= & \phi \mathbb{E}\left\{P_{\mathrm{r}_{\mathrm{u}_{0}, \mathrm{k}}}\right\}=\phi \frac{P_{\mathrm{t}, B_{k_{x^{*}}}} N_{k} L_{0}}{\Pi_{k}}\left(d^{-\eta_{k}} \Psi_{1}+\Psi_{2}\right) \\
& +\phi \frac{L_{0}}{\Pi_{k}} \sum_{j=1}^{K} 2 \pi P_{\mathrm{t}, j} \lambda_{j}\left(\Psi_{3}+\left(\eta_{j}-2\right)^{-1} \Psi_{4}\right),
\end{aligned}
$$

where

$$
\begin{aligned}
\Psi_{1}= & \int_{0}^{d} x \exp \left\{-\sum_{j_{1}=1}^{K} \zeta_{k, j_{1}} x^{2 \eta_{k} / \eta_{j_{1}}}\right\} d x, \\
\Psi_{2}= & \int_{d}^{\infty} x^{-\left(\eta_{k}-1\right)} \exp \left\{-\sum_{j_{2}=1}^{K} \zeta_{k, j_{2}} x^{2 \eta_{k} / \eta_{j_{2}}}\right\} d x, \\
\Psi_{3}= & \int_{0}^{\chi_{j, k}} \frac{x}{2 d^{\eta_{j}}}\left(\frac{\eta_{j} d^{2}}{\left(\eta_{j}-2\right)}-\rho_{j, k}{ }^{2 / \eta_{j}} x^{2 \eta_{j} / \eta_{k}}\right) \\
& \exp \left\{-\sum_{j_{3}=1}^{K} \zeta_{k, j_{3}} x^{2 \eta_{k} / \eta_{j_{3}}}\right\} d x, \\
\Psi_{4}= & \int_{\chi_{j, k}}^{\infty} \frac{x \exp \left\{-\sum_{j_{4}=1}^{K} \zeta_{k, j_{4}} x^{2 \eta_{k} / \eta_{j_{4}}}\right\}}{\left(\rho_{j, k}{ }^{1 / \eta_{j}} x^{\eta_{k} / \eta_{j}}\right)^{\eta_{j}-2}} d x,
\end{aligned}
$$

$$
\begin{gathered}
\chi_{j, k}=d^{\eta_{j} / \eta_{k}} \rho_{j, k}{ }^{-1 / \eta_{k}}, \\
\Pi_{k}=\int_{0}^{\infty} r \exp \left\{-\sum_{j_{0}=1}^{K} \zeta_{k, j_{0}} r^{2 \eta_{k} / \eta_{j_{0}}}\right\} d r \\
\zeta_{k, j}=\pi \lambda_{j} \rho_{j, k}{ }^{2 / \eta_{j}},
\end{gathered}
$$

and

$$
\rho_{j, k}=P_{\mathrm{t}, j} B_{j} / P_{\mathrm{t}, k} B_{k} .
$$

Proof. See Appendix A.

In the following, we present the maximum transmit power at the typical MT in HCNs for the special case of single antenna BSs and equal path loss exponent in each tier.

Corollary 1. With $N_{k}=1$ and $\left\{\eta_{k}\right\}=\eta$, the maximum transmit power at the typical MT associated with the kth tier reduces to

$$
\begin{aligned}
P_{\mathrm{t}_{\mathrm{u}}, k}= & \phi P_{\mathrm{t}, k} N_{k} L_{0}\left[d^{-\eta} \Lambda_{1}+\Lambda_{2}\right]+ \\
& \phi L_{0} \sum_{j=1}^{K} \pi P_{\mathrm{t}, j} \lambda_{j}\left[\Lambda_{3}+(\eta-2)^{-1} \Lambda_{4}\right],
\end{aligned}
$$

where

$$
\begin{aligned}
& \Lambda_{1}=1-\exp \left\{-d^{2} \varpi_{k}\right\}, \\
& \Lambda_{2}=\left(\varpi_{k}\right)^{\eta / 4} d^{-\eta / 2} \exp \left\{-d^{2} \varpi_{k} / 2\right\} W_{-\eta / 4,1 / 2(1-\eta / 2)}\left(d^{2} \varpi_{k}\right),
\end{aligned}
$$

$$
\begin{aligned}
\Lambda_{3}= & \frac{\eta}{\eta-2} d^{(2-\eta)}\left(1-\exp \left\{-\varpi_{k} d^{2} \rho_{j, k}{ }^{-2}\right\}\right)-\frac{\rho_{j, k}{ }^{2 / \eta}}{d^{\eta} \varpi_{k}} \\
& \Upsilon\left(2, \varpi_{k} d^{2} \rho_{j, k}{ }^{-2 / \eta}\right), \\
\Lambda_{4}= & 2 \rho_{j, k}{ }^{-1 / \eta-1 / 2} \varpi_{k}{ }^{7 / 2-3 \eta / 4} d^{-\eta / 2+1} \\
& \exp \left\{-d^{2} \rho_{j, k}{ }^{-2 / \eta} \varpi_{k} / 2\right\}, \\
\varpi_{k}= & \sum_{j_{1}=1}^{K} \zeta_{k, j_{1}}=\sum_{j_{1}=1}^{K} \pi \lambda_{j_{1}}\left(P_{\mathrm{t}, j_{1}} B_{j_{1}} / P_{\mathrm{t}, k} B_{k}\right)^{2 / \eta},
\end{aligned}
$$

and $W_{\lambda, \mu}(z)$ is Whittaker function [39].

\section{System-LeVel Performance Evaluation of UPLINK INFORMATION TRANSMISSION}

In the UL transmission, we focus on the impact of DL wireless power transfer, and the power control is not considered in the UL transmission [23]. We characterize two important performance metrics of UL transmission in HCNs, namely, the UL outage probability per MT and the UL average ergodic rate per MT.

\section{A. Uplink Outage Probability}

With RF wireless power transfer in HCNs, the UL outage probability is a crucial metric, as we will see from (21), and it reflects the energy harvesting efficiency of DL power transfer and the reliability of UL information delivery. Here, the outage occurs when the data rate during a communication block $T$ is below a predetermined target data rate $R_{S}$. Using the law of 
total probability, the UL outage probability of a random MT is given by [25]

$$
P_{\text {out }}\left(R_{S}\right)=\sum_{k=1}^{K} J_{k} P_{\text {out }, k}\left(R_{S}\right)
$$

where $J_{k}$ is the probability that the typical MT is associated with the $k$ th tier, and $P_{\text {out }, k}\left(R_{S}\right)$ is the UL outage probability of a typical MT associated with the $k$ th tier.

In (20), the UL outage probability of a typical MT that is associated with its serving BS in the $k$ th tier is defined as

$$
\begin{aligned}
& P_{\text {out }, k}\left(R_{S}\right)=\mathbb{E}_{\| \mathbf{x}_{\mathrm{u}_{0}, S_{k^{*}}}} \|\left\{\operatorname { P r } \left(\frac{(1-\alpha) T}{T}\right.\right. \\
& \left.\left.\ln \left(1+\operatorname{SINR}_{k}\left(\left\|\mathbf{x}_{\mathrm{u}_{0}, S_{k^{*}}}\right\|\right)\right) \leq R_{S}\right)\right\} \\
& =\mathbb{E}_{\| \mathbf{x}_{\mathrm{x}_{0}, S_{k^{*}}}} \|\left\{\operatorname{Pr}\left(\operatorname{SINR}_{k}\left(\left\|\mathbf{x}_{\mathrm{u}_{0}, S_{k_{x^{*}}}}\right\|\right) \leq \gamma_{t h}\right)\right\},
\end{aligned}
$$

where the SINR threshold is

$$
\gamma_{t h}=e^{R_{S} /(1-\alpha)}-1
$$

In (20), the probability that the typical MT is associated with a $\mathrm{BS}$ in the $k$ th tier is given by

$$
J_{k}=2 \pi \lambda_{k} \int_{0}^{\infty} r \exp \left\{-\sum_{j_{0}=1}^{K} \zeta_{k, j_{0}} r^{2 \eta_{k} / \eta_{j_{0}}}\right\} d r
$$

where $\zeta_{k, j}$ is given in (12).

Theorem 2. The UL outage probability of a typical MT in the $k$ th tier is derived as

$P_{\text {out }, k}\left(R_{S}\right)=1-\frac{1}{\Pi_{k}}\left[\Theta_{1}+\sum_{m=1}^{N-1} \frac{\phi^{-m}}{(-1)^{m}} \sum \frac{1}{\prod_{i=1}^{m} n_{i} ! i !^{n_{i}}} \Theta_{2}\right]$,

where

$$
\begin{aligned}
\Theta_{1}= & \int_{0}^{\infty} x \exp \left\{-\sigma^{2} \tau_{k} \gamma_{t h} x^{\eta_{k}}-\sum_{j=1}^{K} x^{\frac{2 \eta_{k}}{\eta_{j}}}\right. \\
& \left.\left(\vartheta_{k, j}\left(\gamma_{t h}\right)^{\frac{2}{\eta_{j}}}+\zeta_{k, j}\right)\right\} d x \\
\Theta_{2}= & \int_{0}^{\infty} x \exp \left\{-\sigma^{2} \tau_{k} \gamma_{t h} x^{\eta_{k}}-\sum_{j=1}^{K} x^{\frac{2 \eta_{k}}{\eta_{j}}}\right. \\
& \left.\left(\vartheta_{k, j}\left(\gamma_{t h}\right)^{\frac{2}{\eta_{j}}}+\zeta_{k, j}\right)\right\} \\
& \left(-\sigma^{2} \tau_{k} \phi \gamma_{t h} x^{\eta_{k}}-\sum_{j=1}^{K} \vartheta_{k, j}\left(\gamma_{t h}\right)^{\frac{2}{\eta_{j}}} \frac{2}{\eta_{j}} \phi x^{\frac{2 \eta_{k}}{\eta_{j}}}\right)^{n_{1}} \\
& \prod_{l=2}^{m}\left(-\sum_{j=1}^{K} \vartheta_{k, j}\left(\gamma_{t h}\right)^{\frac{2}{\eta_{j}}} \prod_{i=0}^{l-1}\left(\frac{2}{\eta_{j}}-i\right) \phi^{l} x^{\frac{2 \eta_{k}}{\eta_{j}}}\right)^{n_{l}} d x
\end{aligned}
$$

$$
\tau_{k}=\left(\phi \mathbb{E}\left\{P_{r_{\mathrm{u}}, k}\right\} L_{0}\right)^{-1},
$$

$$
\begin{aligned}
\vartheta_{k, j}= & \pi \lambda_{j}\left(\mathbb{E}\left\{P_{r_{\mathrm{u}}, j}\right\} / \mathbb{E}\left\{P_{r_{\mathrm{u}}, k}\right\}\right)^{\frac{2}{\eta_{j}}} \Gamma\left(1+\frac{2}{\eta_{j}}\right) \\
& \Gamma\left(1-\frac{2}{\eta_{j}}\right),
\end{aligned}
$$

and the summation $\sum$ is over all m-tuples of nonegative integers $\left(n_{1}, \ldots, n_{m}\right)$ satisfying the constraint $1 \cdot n_{1}+2 \cdot n_{2}+$ $3 \cdot n_{3}+\cdots m \cdot n_{m}=m$. In (24), $\zeta_{k, j}, \Pi_{k}, \gamma_{t h}$, and $\mathbb{E}\left\{P_{\mathrm{r}_{\mathrm{u}}, k}\right\}$ are given in (12), (11), (22), and (5), respectively.

\section{Proof. See Appendix B.}

1) Interference-Limited Scenario: For the UL transmission of HCNs with high density of high transmit power MTs, the interference power from the interfering MTs in each tier dominates the performance, and as such the thermal noise is ignored. In the following, we present the UL outage probability in the interference-limited scenario.

Corollary 2. With $\left\{\eta_{k}\right\}=\eta$ and $\sigma^{2}=0$, the UL outage probability of a typical MT associated with the kth tier is derived as

$$
\begin{aligned}
& P_{\text {out }, k}\left(R_{S}\right)= \\
& 1-\left(1+\varpi_{k}{ }^{-1} \sum_{j=1}^{K} \vartheta_{k, j}\left(\gamma_{t h}\right)^{\frac{2}{\eta}}\right)^{-1}-\sum_{m=1}^{N-1} \frac{1}{(-1)^{m}} \\
& \sum \frac{\varpi_{k}}{\prod_{i=1}^{m} n_{i} ! i !^{n_{i}}} \prod_{l=1}^{m}\left(-\sum_{j=1}^{K} \vartheta_{k, j}\left(\gamma_{t h}\right)^{\frac{2}{\eta}} \prod_{i=0}^{l-1}\left(\frac{2}{\eta_{j}}-i\right)\right)^{n_{l}} \\
& \Gamma\left(\sum_{l=1}^{m} n_{l}+1\right)\left(\varpi_{k}+\sum_{j=1}^{K} \vartheta_{k, j}\left(\gamma_{t h}\right)^{\frac{2}{\eta}}\right)^{-\left(1+\sum_{l=1}^{m} n_{l}\right)}, \text { (29) }
\end{aligned}
$$

where $\varpi_{k}$ and $\vartheta_{k, j}$ are given in (19) and (28). When each BS is equipped with single antenna, (29) reduces to (30).

$$
P_{\text {out }, k}\left(R_{S}\right)=\frac{1}{\frac{\varpi_{k}}{\sum_{j=1}^{K} \vartheta_{k, j}}\left(2^{R_{S} /(1-\alpha)}-1\right)^{-\frac{2}{\eta}}+1},
$$

where

$$
\begin{gathered}
\frac{\varpi_{k}}{\sum_{j=1}^{K} \vartheta_{k, j}}=\sum_{j=1}^{K}\left[\frac{\left(P_{\mathrm{t}, j} B_{j} / P_{\mathrm{t}, k} B_{k}\right)}{\left(\mathbb{E}\left\{P_{r_{\mathrm{u}}, j}\right\} / \mathbb{E}\left\{P_{r_{\mathrm{u}}, k}\right\}\right)}\right]^{2 / \eta} \\
(\Gamma(1+2 / \eta) \Gamma(1-2 / \eta))^{-1}
\end{gathered}
$$

and $\gamma_{t h}$ is given in (22).

According to (29) and (30), we offer the following remarks:

Remark 1. Intrinsically, the UL outage probability per MT is determined by the density of BSs in each tier, because the average received power at the MT in (31) is determined by the density of BSs as shown in (5).

Remark 2. In the interference-limited scenario, the UL outage probability per MT is independent of the RF-to-DC conversion efficiency factor $\mu$. This is attributed to the fact that with omitting $\left(\delta^{2}=0\right)$, the term $\mu$ in (4) is counteracted, as such the UL SINR is independent of $\mu$. 
2) Noise-Limited Scenario: In the DL power transfer phase, the MTs may harvest small amount of energy for UL transmission. For the UL transmission of HCNs with low density of low transmit power MTs, the thermal noise dominates the performance, due to the low interference power from the interfering MTs in each tier (i.e., $I_{U}$ in (4)). In the following, we ignore the intercell interference, and present the UL outage probability in the noise-limited scenario.

Corollary 3. With $\left\{\eta_{k}\right\}=\eta$ and $I_{U}=0$, the UL outage probability of a typical MT associated with the kth tier is derived as

$$
\begin{aligned}
P_{\text {out }, k}\left(R_{S}\right)= & 1-\frac{1}{\Pi_{k}} \sum_{m=0}^{N-1} \frac{1}{m !}\left(\sigma^{2} \gamma_{t h} \tau_{k}\right)^{m} \int_{0}^{\infty} r^{\eta m+1} \\
& \exp \left(-\sigma^{2} \gamma_{t h} \tau_{k} r^{\eta}-\varpi_{k} r^{2}\right) d r
\end{aligned}
$$

where $\varpi_{k}, \tau_{k}$, and $\gamma_{t h}$ are given in (19), (27), and (22), respectively. When each $B S$ is equipped with single antenna, (32) reduces to (33).

$$
\begin{aligned}
P_{\text {out }, k}\left(R_{S}\right)= & -\frac{1}{2 \Pi_{k}} \int_{0}^{\infty} \exp \left(-\sigma^{2} \gamma_{t h}\left(\phi \mathbb{E}\left\{P_{r_{\mathrm{u}_{0}}, k}\right\} L_{0}\right)^{-1}\right. \\
& \left.r^{\eta / 2}-\varpi_{k} r\right) d r
\end{aligned}
$$

where

$$
\mathbb{E}\left\{P_{\mathrm{r}_{\mathrm{u}}, k}\right\}=\frac{P_{\mathrm{t}, B_{k_{x^{*}}}} N_{k} L_{0}}{\Pi_{k}}\left(d^{-\eta_{k}} \Psi_{1}+\Psi_{2}\right),
$$

$\varpi_{k}$ and $\gamma_{t h}$ are given in (19) and (22), respectively.

Remark 3. We find that in the noise-limited scenario, the UL outage probability decreases with increasing the RF-to-DC conversion efficiency.

\section{B. Uplink Average Ergodic Rate}

In $K$-tier HCNs, the UL average ergodic rate is an important performance indicator for spectral efficiency. Similar to the definition of the UL outage probability of a random MT in HCNs in (20), the UL average ergodic rate of a random MT in $\mathrm{K}$-tier $\mathrm{HCN}$ is given by

$$
R=\sum_{k=1}^{K} J_{k} R_{k}
$$

where $J_{k}$ is given in (23). In (35), $R_{k}$ is the UL average ergodic rate of a typical MT associated with the $k$ th tier, which represents the average data rate of a random MT associated with the $k$ th tier during a communication block $T$. In each tier, one MT per cell is active.

For a typical MT at a distance $\left\|\mathbf{x}_{\mathrm{u}_{0}, B_{k^{*}}}\right\|$ from the serving $\mathrm{BS}$, the UL average ergodic rate of a typical MT associated with the $k$ th tier is defined as

$$
\begin{aligned}
R_{k}= & \mathbb{E}_{\left\|\mathbf{x}_{\mathrm{u}_{0}, S_{k^{*}}}\right\|}\left[\mathbb { E } _ { S I N R _ { k } } \left[\frac{(1-\alpha) T}{T}\right.\right. \\
& \left.\left.\ln \left(1+S I N R_{k}\left(\left\|\mathbf{x}_{\mathrm{u}_{0}, S_{k_{x^{*}}}}\right\|\right)\right)\right]\right] .
\end{aligned}
$$

We derive the UL average ergodic rate of a typical MT associated with the $k$ th tier in the following theorem.
Theorem 3. The UL average ergodic rate of a typical MT associated with the $k$ th tier is derived as

$$
R_{k}=\frac{(1-\alpha)}{\Pi_{k}}\left[\mathrm{~T}_{1}-\sum_{m=1}^{N-1} \frac{\phi^{-m}}{(-1)^{m}} \sum \frac{1}{\prod_{i=1}^{m} n_{i} ! i !^{n_{i}}} \mathrm{~T}_{2}\right] \text {, }
$$

where

$$
\begin{aligned}
\mathrm{T}_{1}= & \int_{0}^{\infty} \int_{0}^{\infty} \frac{r}{1+x} \exp \left\{-\sigma^{2} \tau_{k} x r^{\eta_{k}}-\sum_{j=1}^{K}\left(\vartheta_{k, j} x^{\frac{2}{\eta_{j}}}+\zeta_{k, j}\right)\right. \\
& \left.r^{\frac{2 \eta_{k}}{\eta_{j}}}\right\} d x d r
\end{aligned}
$$

$$
\begin{aligned}
\mathrm{T}_{2}= & \int_{0}^{\infty} \int_{0}^{\infty} \exp \left\{-\sigma^{2} \tau_{k} x r^{\eta_{k}}-\sum_{j=1}^{K}\left(\vartheta_{k, j} x^{\frac{2}{\eta_{j}}}+\zeta_{k, j}\right) r^{\frac{2 \eta_{k}}{\eta_{j}}}\right\} \\
& \frac{r}{1+x}\left(-\sigma^{2} \tau_{k} \phi x r^{\eta_{k}}-\sum_{j=1}^{K} \vartheta_{k, j} \frac{2}{\eta_{j}} \phi x^{\frac{2}{\eta_{j}}} r^{\frac{2 \eta_{k}}{\eta_{j}}}\right)^{n_{1}} \\
& \prod_{l=2}^{m}\left(-\sum_{j=1}^{K} \vartheta_{k, j} \prod_{i=0}^{l-1}\left(\frac{2}{\eta_{j}}-i\right) \phi^{l} x^{\frac{2}{\eta_{j}}} r^{\frac{2 \eta_{k}}{\eta_{j}}}\right)^{n_{l}} d x d r
\end{aligned}
$$

and, $\vartheta_{k, j}, \tau_{k}, \zeta_{k, j}$ and $\mathbb{E}\left\{P_{\mathrm{r}_{\mathrm{u}}, k}\right\}$ are given in (28), (27), (12) and (5), respectively.

Proof. See Appendix C.

1) Interference-Limited Scenario: In the following, we present the UL average ergodic rate of a typical MT associated with the $k$ th tier in HCNs with equal path loss exponent in each tier in the interference-limited scenario.

Corollary 4. With $\left\{\eta_{k}\right\}=\eta$ and $\sigma^{2}=0$, the UL average ergodic rate of a typical MT associated with the kth tier is derived as

$$
\begin{aligned}
R_{k}= & (1-\alpha)\left[\Xi_{1}-\frac{1}{\Pi_{k}} \sum_{m=1}^{N-1} \frac{1}{(-1)^{m}} \sum \frac{1}{\prod_{i=1}^{m} n_{i} ! i !^{n_{i}}}\right. \\
& \left.\prod_{l=1}^{m} \Gamma\left(\sum_{l=1}^{m} n_{l}+1\right) \Xi_{2}\right],
\end{aligned}
$$

where

$$
\Xi_{1}=\int_{0}^{\infty} \varpi_{k}\left[(1+x)\left(\varpi_{k}+\sum_{j=1}^{K} \vartheta_{k, j} x^{\frac{2}{\eta}}\right)\right]^{-1} d x
$$

and

$$
\begin{aligned}
\Xi_{2}= & \int_{0}^{\infty} \varpi_{k}\left(-\sum_{j=1}^{K} \vartheta_{k, j} \prod_{i=0}^{l-1}\left(\frac{2}{\eta}-i\right) x^{\frac{2}{\eta}}\right)^{n_{l}} \\
& {\left[(1+x)\left(\varpi_{k}+\sum_{j=1}^{K} \vartheta_{k, j} x^{\frac{2}{\eta}}\right)^{\left(\sum_{l=1}^{m} n_{l}+1\right)}\right]^{-1} d x . }
\end{aligned}
$$

When each BS is equipped with single antenna, (40) reduces to (43).

$$
R_{k}=(1-\alpha) \int_{0}^{\infty}\left[(1+x)\left(1+x^{\frac{2}{\eta}} \frac{\sum_{j=1}^{K} \vartheta_{k, j}}{\varpi_{k}}\right)\right]^{-1} d x
$$


Remark 4. We find that the UL average ergodic rate in the interference-limited scenario is independent of the RF-to-DC conversion efficiency. This can be seen from (4) where $\mu$ in the $S I N R_{k}$ disappear in the interference-limited regime with $\sigma^{2}=0$.

2) Noise-Limited Scenario: In the following, we present the UL average ergodic rate of a typical MT associated with the $k$ th tier in HCNs with equal path loss exponent in each tier in the noise-limited scenario.

Corollary 5. With $\left\{\eta_{k}\right\}=\eta$ and $I_{U}=0$, the UL average ergodic rate of a typical MT associated with the kth tier is derived as

$$
\begin{aligned}
R_{k}= & \frac{(1-\alpha)}{\Pi_{k}} \sum_{m=0}^{N-1} \frac{1}{m !} \int_{0}^{\infty} \int_{0}^{\infty}\left[\sigma^{2}\left(e^{t}-1\right) \tau_{k}\right]^{m} r^{\eta m+1} \\
& \exp \left(-\sigma^{2}\left(e^{t}-1\right) \tau_{k} r^{\eta}-r^{2} \varpi_{k}\right) d t d r
\end{aligned}
$$

where $\varpi_{k}$ and $\tau_{k}$ are given in (19) and (27), respectively. When each BS is equipped with single antenna, (44) reduces to (45).

$$
\begin{aligned}
R_{k}= & \frac{(1-\alpha)}{2 \Pi_{k}} \int_{0}^{\infty} \int_{0}^{\infty} \exp \left(-\sigma^{2}\left(e^{t}-1\right)\left(\phi E\left\{P_{r_{\mathrm{u}_{0}}, k}\right\} L_{0}\right)^{-1}\right. \\
& \left.r^{\eta / 2}-r \varpi_{k}\right) d t d r
\end{aligned}
$$

where $\varpi_{k}$ is given in (19).

Remark 5. We notice that in the noise-limited scenario, the UL average ergodic rate increases with increasing the RF-to-DC conversion efficiency.

\section{As ymptotic PERFormance EVALUATION}

In this section, we analyze the asymptotic performance of $K$-tier HCNs in which each BS is equipped with the large antenna array. We examine the asymptotic average received power, UL outage probability, and UL average ergodic rate per MT, as the number of antennas at each BS goes to infinity. To perform the asymptotic analysis, we assume that all BSs in the network are equipped with the same number of antennas $N_{k}=N$. According to the law of large numbers, we have

$$
\lim _{N \rightarrow \infty}\left\|\mathbf{h}_{\mathrm{u}_{0}, S_{k_{x}}}\right\|^{2}=N
$$

\section{A. Downlink Power Transfer}

In the DL HCNs with sufficiently large number of antennas $N$ at the BS, we rewrite the received power at the typical MT associated with the $k$ th tier using (46) as

$$
P_{\mathrm{r}_{\mathrm{u}_{0}, \mathrm{k}}}^{\infty}=\underbrace{P_{\mathrm{t}, S_{k_{x^{*}}}} N L_{0}\left(\max \left\{\left\|\mathbf{x}_{S_{k_{x^{*}}}}\right\|, d\right\}\right)^{-\eta_{k}}}_{I_{S_{k_{x^{*}}}}} .
$$

Theorem 4 . When $N$ is sufficiently large, the average received power at the typical MT in the $k$ th tier with BRP cell association rule is derived as

$$
\begin{aligned}
& \mathbb{E}\left\{P_{\mathrm{r}_{\mathrm{u}}, k}^{\infty}\right\}= \\
& \quad \frac{P_{\mathrm{t}, S_{k_{x^{*}}}} N L_{0}}{\Pi_{k}}\left[d^{-\eta_{k}} \int_{0}^{d} x \exp \left\{-\sum_{j_{1}=1}^{K} \zeta_{k, j_{1}} x^{2 \eta_{k} / \eta_{j_{1}}}\right\} d x\right. \\
& \left.\quad+\int_{d}^{\infty} x^{-\left(\eta_{k}-1\right)} \exp \left\{-\sum_{j_{2}=1}^{K} \zeta_{k, j_{2}} x^{2 \eta_{k} / \eta_{j_{2}}}\right\} d x\right] .
\end{aligned}
$$

We can see that the average received power proportionally increases with $N$, i.e., $\mathbb{E}\left\{P_{\mathrm{r}_{\mathrm{u}}, k}^{\infty}\right\} \propto N$.

\section{B. Uplink Information Transmission}

When the number of antennas at each BS is sufficiently large, by using (46), the SINR of the UL transmission with $\mathrm{MRC}$ at the typical BS is given by

$$
S I N R_{k}^{\infty}=\frac{\phi \mathbb{E}\left\{P_{r_{\mathrm{u}_{0}}, k}^{\infty}\right\} N L_{0}\left\|x_{\mathrm{u}, S_{k_{x^{*}}}}\right\|^{-\eta_{k}}}{I_{U}^{\infty}+\delta^{2}},
$$

where $I_{U}^{\infty}=\sum_{j=1}^{K} I_{U, j}^{\infty}, I_{U, j}^{\infty}=\sum_{\mathrm{u}_{x, j} \in \tilde{\Phi}_{j} \backslash \mathrm{u}_{0}} \phi \mathbb{E}\left\{P_{r_{\mathrm{u}}, j}^{\infty}\right\}$

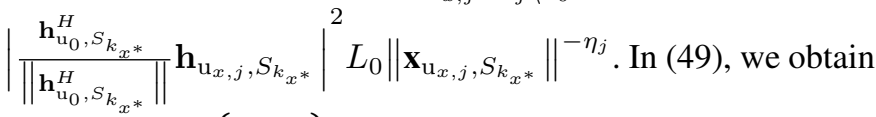
$S I N R_{k}^{\infty} \propto \mathbb{E}\left\{P_{\mathrm{r}_{\mathrm{u}}, k}^{\infty}\right\} \propto N$.

1) Asymptotic Uplink Outage Probability: We present the asymptotic UL outage probability of a typical MT associated with the $k$ th tier in the following theorem.

Theorem 5. As $N \rightarrow \infty$, the UL outage probability of a typical MT associated with the $k$ th tier is derived as

$P_{\text {out }, k}^{\infty}\left(R_{S}\right)=$

$\frac{1}{2}+\frac{1}{\pi \Pi_{k}} \int_{0}^{\infty} \int_{0}^{\infty} \operatorname{Im}\left[\exp \left\{-j w\left(N_{k} r^{-\eta_{k}}\left(\tau_{k} \gamma_{t h}\right)^{-1}-\sigma^{2}\right)\right\}\right.$

$\left.\psi^{*}(w)\right] \frac{1}{w} d w r \exp \left\{-\sum_{j_{0}=1}^{K} \zeta_{k, j_{0}} r^{2 \eta_{k} / \eta_{j_{0}}}\right\} d r$,

where $\psi^{*}(w)$ is the conjugate of the characteristic function,

$$
\begin{aligned}
\psi(w)= & \exp \left\{-\sum_{j=1}^{K} \lambda_{j} \pi\left(\phi \mathbb{E}\left\{P_{r_{\mathrm{u}}, j}\right\} L_{0}\right)^{\frac{2}{\eta_{j}}} \Gamma\left(1+\frac{2}{\eta_{j}}\right)\right. \\
& \left.\Gamma\left(1-\frac{2}{\eta_{j}}\right)(j w)^{\frac{2}{\eta_{j}}}\right\},
\end{aligned}
$$

and $\gamma_{t h}$ is given in (22).

Proof. See Appendix D.

Due to the conjugate of the characteristic function and double integrations in (50), we can not observe the impact of system parameters on the UL performance. However, it is a much simplified expression compared with (24), which has different sets of summation that change according to $N$. 


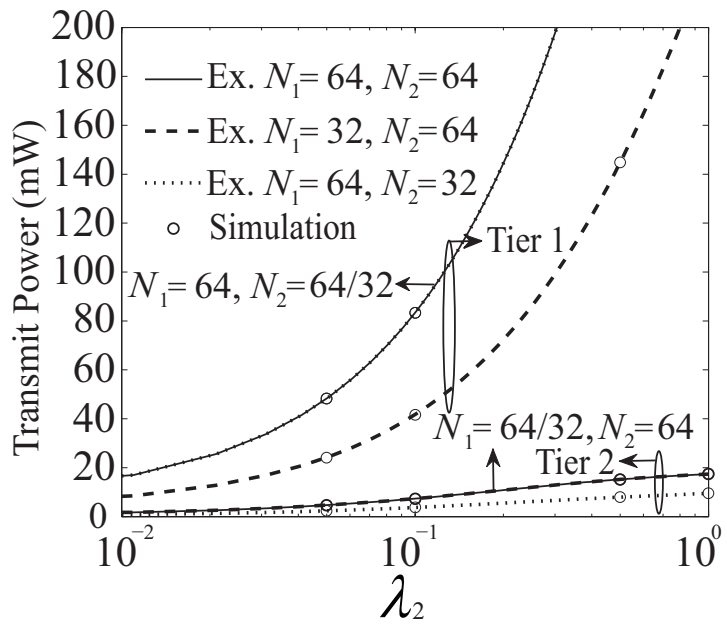

Fig. 1. Impact of number of BS antennas and picocell BS density on the maximum transmit power.

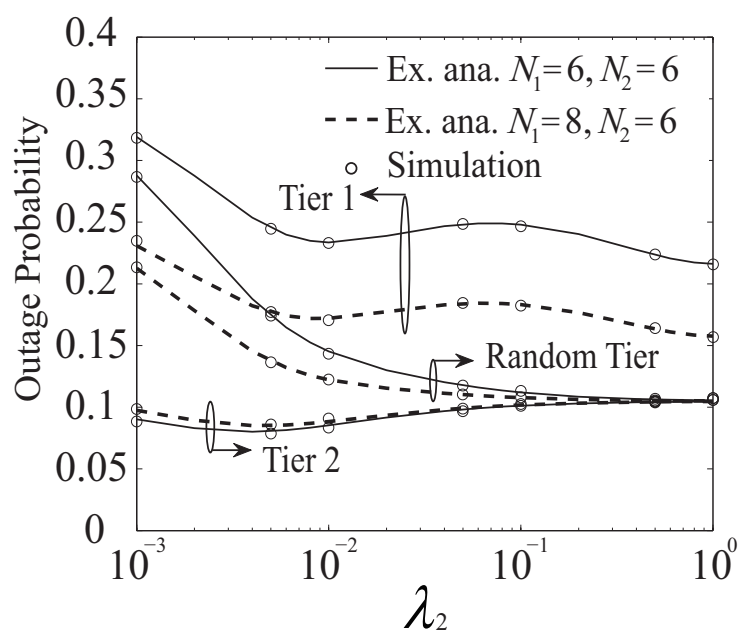

Fig. 2. Impact of number of BS antennas and picocell BS density on the outage probability.

2) Asymptotic Uplink Average Ergodic Rate: We present the asymptotic UL average ergodic rate of a typical MT associated with the $k$ th tier, when $N$ is sufficiently large, in the following theorem.

Theorem 6. As $N \rightarrow \infty$, the UL average ergodic rate of a typical MT associated with the $k$ th tier is derived as

$$
\begin{aligned}
& R_{k}^{\infty}=(1-\alpha)\left[\frac{1}{2}-\frac{1}{\pi \Pi_{k}} \int_{0}^{\infty} \int_{0}^{\infty} \int_{0}^{\infty} \operatorname{Im}[\exp \{-j w\right. \\
& \left.\left.\left(N_{k} r^{-\eta_{k}}\left(\tau_{k} t\right)^{-1}-\sigma^{2}\right)\right\} \psi^{*}(w)\right] \exp \left\{-\sum_{j_{0}=1}^{K} \zeta_{k, j_{0}} r^{2 \eta_{k} / \eta_{j_{0}}}\right\} \\
& \left.\frac{r}{w(t+1)} d w d t d r\right]
\end{aligned}
$$

where $\psi(w)$ is given in (51).

Even though the asymptotic UL average ergodic rate is composed of triple integrations, it is in a much simpler form

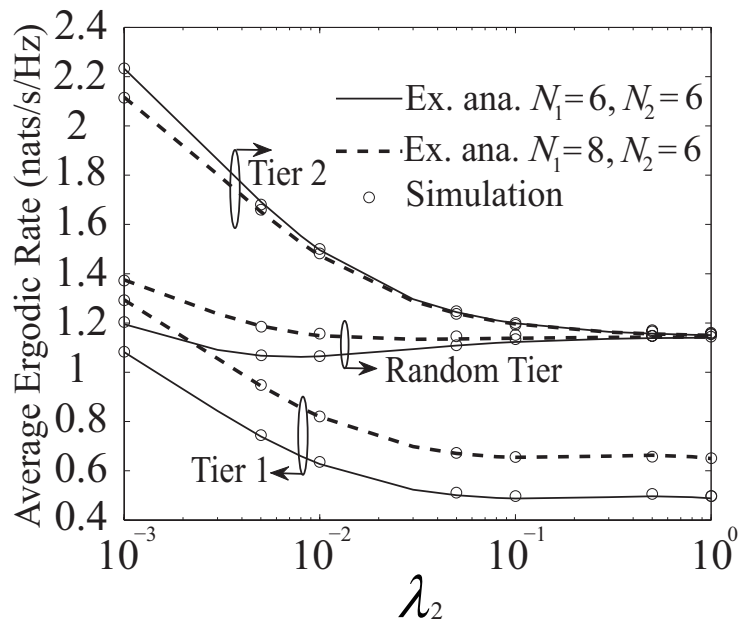

Fig. 3. Impact of number of BS antennas and picocell BS density on the average ergodic rate.

with shorter computation time compared to the exact UL average ergodic rate in (37).

\section{NUMERICAL RESULTS}

In this section, the exact maximum transmit power, the UL outage probability, and the UL average ergodic rate per MT are plotted using (5), (24), and (37), respectively, and the asymptotic UL outage probability and UL average ergodic rate per MT are plotted using (50) and (52), respectively. The analytical results are validated by Monte Carlo simulations as shown in each figure. In all the figures, we set the path loss at 1 meter of $L_{0}=-38.5 \mathrm{~dB}$, and the thermal noise $\sigma^{2}=-104 \mathrm{~dB}$ for $10 \mathrm{MHz}$ bandwidth. In the figures, "Ex. ana." represents exact analytical plot. Even though the numerical results are given for two-tier HCNs model, it can be easily expanded to $K$-tier HCNs without loss of generality.

\section{A. Impact of Number of BS Antennas and Picocell BS Density}

In this subsection, we examine the effect of the number of antennas at each BS and the density of picocell BSs on the maximum transmit power, the UL outage probability, and the UL average ergodic rate per MT of the proposed model. In Fig. 1, 2, and 3, we set $\eta_{1}=3.8, \eta_{2}=3.5, \lambda_{1}=10^{-3} \mathrm{~m}^{-2}$, $B_{1}=B_{2}=1, P_{t, S_{1}}=46 \mathrm{dBm}, P_{t, S_{2}}=28 \mathrm{dBm}, d=1$, $T=1$, and $R_{s}=0.1$.

Fig. 1 plots the maximum transmit power at the typical MT versus the density of picocell BSs $\lambda_{2}$. We set $\alpha=0.8$, $\mu=0.7$. The following insights are observed: 1) The maximum transmit power at the MT in marcocell or picocell increases with increasing $\lambda_{2}$. This can be explained by (A.2) that increasing $\lambda_{2}$ decreases the distance $\left\|x_{S_{k_{x^{*}}}}\right\|$ between the typical MT and the serving BS. 2) We see that the maximum transmit power at the MT in the marcocell is higher and grows sharper than that at the MT in the picocell. This is due to the fact that the transmit power of the macrocell BS is much higher than that of the picocell BS; and 3) We find that for $N_{1}=64$, increasing the number of antennas at the picocell 


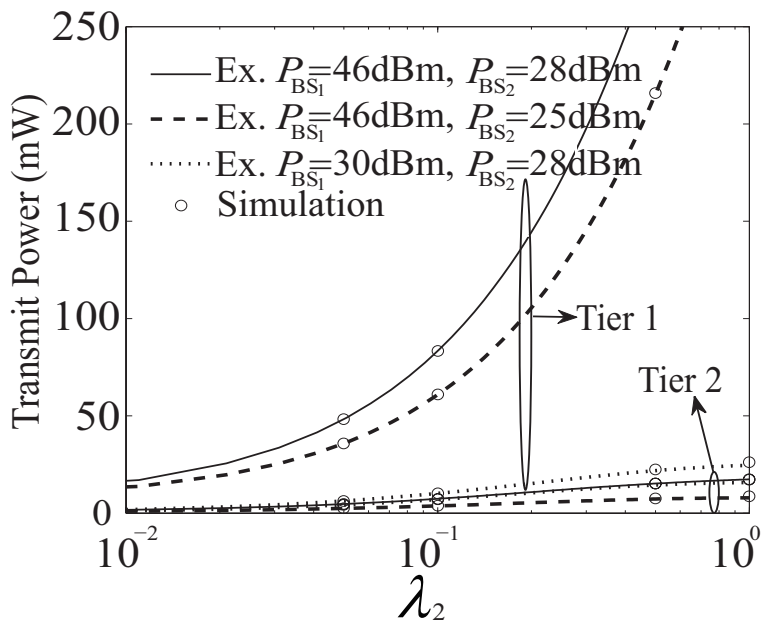

Fig. 4. Impact of BS transmit power on the maximum transmit power.

BS $N_{2}$ has no effect on the maximum transmit power at the MT in the macrocell. Similarly, for $N_{2}=64$, increasing the number of antennas at the macrocell BS $N_{1}$ has no effect on the maximum transmit power at the MT in the picocell. These observations are attributed to the fact that changing the number of antennas at the interfering BS does not change the distribution of $\left|\mathbf{h}_{S_{x} u_{0}} \frac{\mathbf{g}_{S_{x} u_{j}}^{H}}{\left\|\mathbf{g}_{S_{x} u_{j}}\right\|}\right|^{2}$ in (3).

Fig. 2 and Fig. 3 plot the UL outage probability and the UL average ergodic rate per MT versus the density of picocell BSs $\lambda_{2}$. We set $\alpha=0.1$ and $\mu=0.2$. The key observations are as follows: We observe that the UL outage probability and the UL average ergodic rate per MT are not significantly improved with increasing $\lambda_{2}$, which is due to the tradeoff between the benefits from the increased transmit power of MT and the detrimental effect brought by the increased interference from the other MTs in the picocell. We also see that increasing the number of antennas at the macrocell BS improves the UL outage probability and the UL average ergodic rate per random MT.

\section{B. Impact of BS Transmit Power}

In this subsection, we examine the effect of the transmit power at the BS on the maximum transmit power at the MT and the UL outage probability per MT. In Fig. 4 and Fig. 5, we set $\eta_{1}=3.8, \eta_{2}=3.5, \lambda_{1}=10^{-3} \mathrm{~m}^{-2}, B_{1}=B_{2}=1$, $d=1, T=1$, and $R_{s}=0.1$.

Fig. 4 plots the maximum transmit power at the typical MT versus the density of picocell BS $\lambda_{2}$ for various transmit power at the BS. We set $\alpha=0.8$ and $\mu=0.7$. The following insights can be observed: 1) The maximum transmit power can be improved by increasing the transmit power of BS in either tier, which can be shown by (3); and 2) The maximum transmit power of typical MT in macrocell is higher and increases sharper than that of typical MT in picocell, which is due to the dominant effect of the energy harvested from the serving BS using energy beamforming.

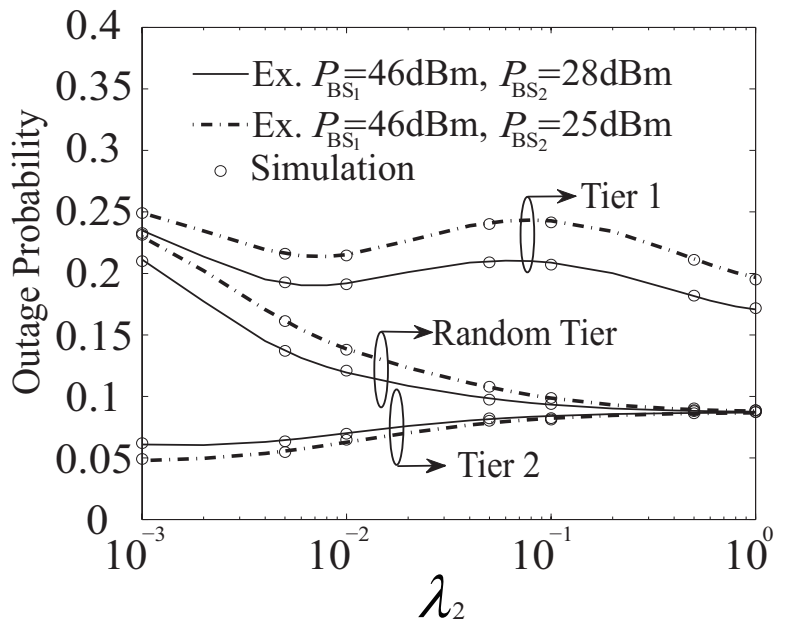

Fig. 5. Impact of BS transmit power on the outage probability.

From Fig. 1 and Fig. 4, we see that the transmit powers from the harvested energy at the macrocell MT and picocell MT increase with the density of picocell BSs, and can be very large $(\geq 20 \mathrm{~mW})$ when deploying more picocell BSs. Note that when the BSs are equipped with large antenna arrays with sharp beamforming, the transmit power values of macrocell MT and picocell MT can be further enlarged. As clarified in [2], in the practical scenario, the power consumption of popular mobile devices ranges from milliwatts for sensors or ZigBee devices to several tens of milliwatts for smartphones. In our model, we show that the transmit power level of MT in both macrocell MT and picocell MT fulfills this practical requirement, especially with the deployment of large antenna array at the BS.

Fig. 5 plots the UL outage probability per MT versus the density of picocell BSs $\lambda_{2}$ for various transmit power at the BS. We set $\alpha=0.1$ and $\mu=0.2$. Following are the observations: Interestingly, for $P_{t, B_{1}}=46 \mathrm{dBm}$, we see that the UL outage probability per picocell MT with $P_{t, B_{2}}=25 \mathrm{dBm}$ outperforms that with $P_{t, B_{2}}=28 \mathrm{dBm}$. This is can be explained by (A.2) that the distance between the typical MT and the associated picocell BS increases with increasing $P_{t, B_{2}}$, and thus the SINR of picocell BS decreases, and the UL performance of picocell BS degrades. We further conclude that the UL outage probability per random MT is not significantly improved with increasing the transmit power of picocell BS.

\section{Impact of Energy Conversion Efficiency and Time Alloca- tion Factor}

In this subsection, we examine the effect of the energy conversion efficiency and the time allocation factor on the UL outage probability, and the UL average ergodic rate per MT. In Fig. 6 and 7, we set $\eta_{1}=3.8, \eta_{2}=3.5, \lambda_{1}=10^{-3} \mathrm{~m}^{-2}$, $\lambda_{2}=2 \times 10^{-3} \mathrm{~m}^{-2}, P_{t, S_{1}}=46 \mathrm{dBm}, P_{t, S_{2}}=28 \mathrm{dBm}$, $B_{1}=B_{2}=1, d=1, T=1, N_{1}=N_{2}=6$, and $R_{s}=0.1$.

Fig. 6 plots the UL outage probability per MT versus the time allocation factor $\alpha$. An interesting observation is that 


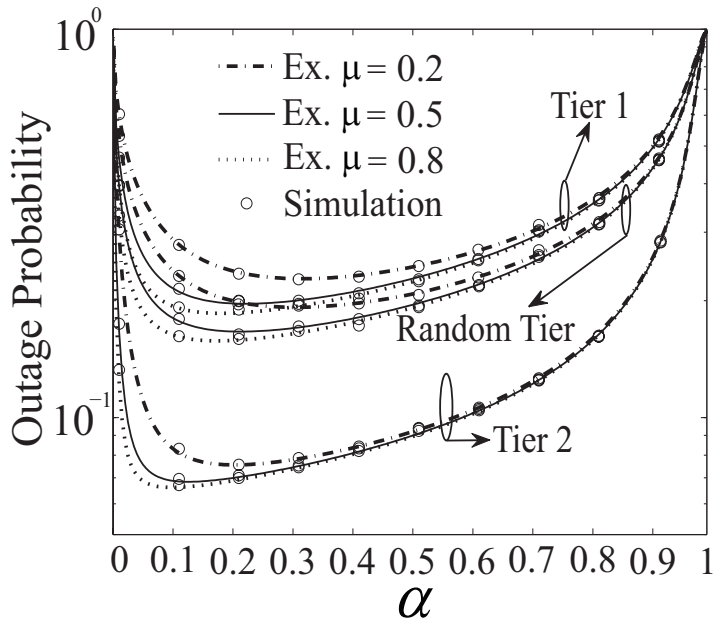

Fig. 6. Impact of energy conversion efficiency and time allocation factor on the outage probability.

the UL outage probability per MT first improves, and then degrades with the increase of $\alpha$. This is because for small $\alpha$, increasing the time allocated to the DL wireless power transfer increases the transmit power at the MT, and improves the UL transmission. However, after an optimal $\alpha$, increasing $\alpha$ decreases the time allocated for the UL information transmission, while the aggregate interference from other MTs increases dramatically due to their high transmit power, thus the UL performance of typical MT degrades. We show that there exists an optimal time allocation factor which achieves the lowest UL outage probability, and undoubtedly the maximum UL outage probability per MT occurs at $\alpha=1$.

Fig. 7 plots the UL average ergodic rate per MT versus the energy conversion efficiency $\mu$. We see that the UL average ergodic rate per MT improves with increasing $\mu$ in noninterference-limited scenario. This is because for small $\mu$, the noise plays a dominant role in the received SINR as shown in (4), and thus the SINR increases with increasing $\mu$ in the noise-limited scenario. However, for large $\mu$, the aggregate interference overtakes the effect of noise, and the SIR dominates the performance in the interference-limited scenario. In this case, the SINR remains almost unchanged with increasing $\mu$, and thus the UL average ergodic rate is almost saturated. In the interference-limited scenario, the UL average ergodic rate per MT is constant for arbitrary $\mu$, which can be explained by Remark 2 .

\section{Impact of BS Large Antenna Array}

In this subsection, we examine the effect of the BS large antenna array on the UL outage probability, and the UL average ergodic rate. In Fig. 8 and 9 , we set $N_{1}=N_{2}$, $\eta_{1}=3.8, \eta_{2}=3.5, \lambda_{1}=10^{-3} \mathrm{~m}^{-2}, \lambda_{2}=2 \times 10^{-3} \mathrm{~m}^{-2}$, $P_{t, S_{1}}=46 \mathrm{dBm}, P_{t, S_{2}}=30 \mathrm{dBm}, B_{1}=B_{2}=1, d=1$, $T=1, R_{s}=1.3, \mu=0.3$, and $\alpha=0.45$.

Figs. 8 and 9 plot the UL outage probability and the UL average ergodic rate per MT versus the number of BS antennas $N$. We see that the asymptotic UL outage probability and

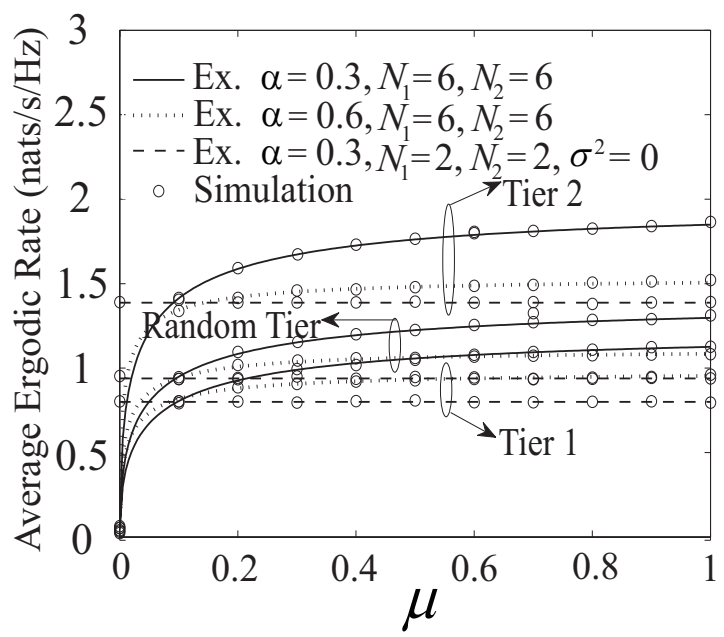

Fig. 7. Impact of energy conversion efficiency and time allocation factor on the average ergodic rate.

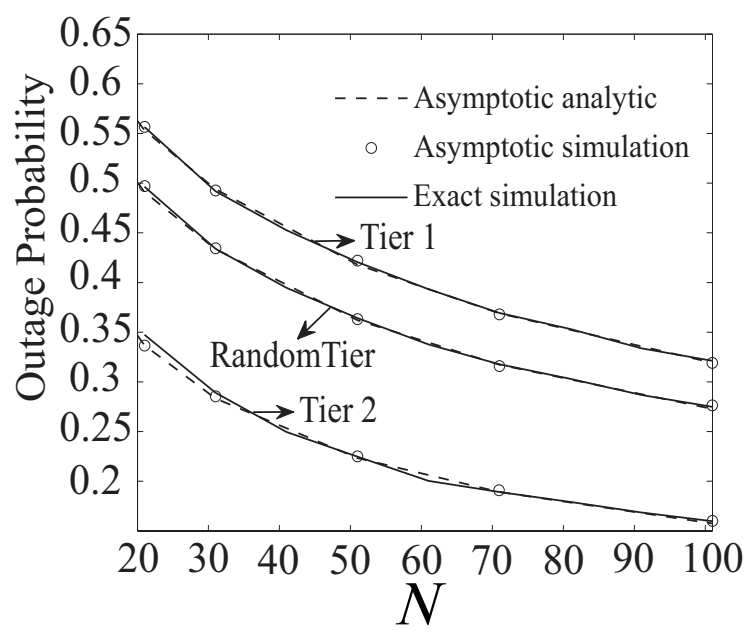

Fig. 8. Impact of BS large antenna array on the outage probability.

asymptotic UL average ergodic rate converge to the exact UL outage probability and exact UL average ergodic rate in the high $N$ regime. We also find that for sufficiently large $N$, the UL performance per MT improves with increasing the number of antennas at each BS, which is consistant with Eq. (49) where $S I N R_{k}^{\infty} \propto N$. This suggests that the UL performance per MT is greatly improved by using the massive antenna arrays at each BS.

\section{E. Discussion}

As specified in 3GPP TS36.101 Section 6.2.3, the minimum and maximum transmit powers of MT in LTE are $-40 \mathrm{dBm}$ $\left(10^{-4} \mathrm{~mW}\right)$ and $23 \mathrm{dBm}(\approx 200 \mathrm{~mW})$, respectively. As showcased in Fig. 1 and Fig. 4, the UEs associated with macrocell BSs can harvest enough energy to achieve the maximum transmit power requirement with large antenna array and high density of picocell BSs, whereas those associated with picocell BS may transmit signals with lower power level. According to 


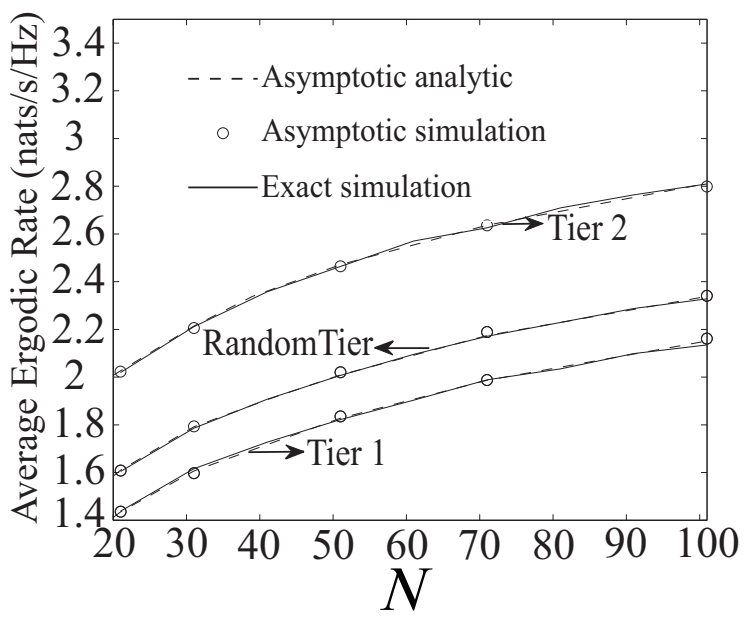

Fig. 9. Impact of BS large antenna array on the average ergodic rate.

$P_{\mathrm{t}_{\mathrm{u}}, k}^{\max }=\mu \frac{\alpha}{1-\alpha} \mathbb{E}\left\{P_{\mathrm{r}_{\mathrm{u}_{0}, \mathrm{k}}}\right\}$, the key for substantially improving the transmit power is to increase the RF-to-DC conversion efficiency and the fraction of time allocated to the DL power transfer.

Based on the fact that different energy levels are harvested from the BSs in different tiers, and the MT is mobile, a potential efficient and enhancement approach for wireless power transfer is to enable the harvested energy stored for several number of time slots in practice. By doing so, the MT has the advantage of harvesting from the direct energy beamforming at both the macrocell $\mathrm{BS}$ and the nearby picocell BS. This can be done by taking into account the battery dynamics using Markov chain modeling. Another extension of this work is to consider the uplink interference management such as power control or interference cancellation in such networks, which can improve the UL outage probability and the UL average ergodic rate in a more energy efficient manner.

\section{CONCLUSION}

We have provided a tractable model for the DL wireless power transfer along with UL information transmission in HCNs. Our analysis presented the analytical expressions for the exact maximum transmit power, the UL outage probability and the UL average ergodic rate per MT, as well as the asymptotic expressions for those important metrics when the number of antennas at BS goes to infinity. The intrinsic relationship between the energy harvested from the BSs in the DL and the information transmission in the UL are well demonstrated using the derived results and simulation results.

Our results revealed that the maximum transmit power at the MT can be improved by adding more picocell BSs, however, the UL outage probability and the UL average ergodic rate per random MT is not significantly improved. In addition, the UL outage probability per MT first improves and then degrades with increasing the time allocation factor, while the UL average ergodic rate improves with increasing the energy conversion efficiency. Our results demonstrated that the DL wireless power transfer can be very efficient to power the uplink information transmission via sharp beamforming with the large antenna array at the BSs.

\section{APPENDIX A \\ A PRoOf OF THEOREM 1}

Based on (3), using $\mathbb{E}\left\{\left\|\mathbf{h}_{S_{k^{*}}}\right\|^{2}\right\}=N_{k}$ and the polarcoordinate system, we first calculate $\mathbb{E}\left\{I_{S_{k_{x}}}\right\}$ as

$$
\begin{aligned}
\mathbb{E}\left\{I_{S_{k^{*}}}\right\}= & \mathbb{E}\left\{P_{\mathrm{t}, S_{k_{x^{*}}}}\left\|\mathbf{h}_{S_{k_{x^{*}}}}\right\|^{2} L_{0}\left(\max \left\{\left\|\mathbf{x}_{S_{k_{x^{*}}}}\right\|, d\right\}\right)^{-\eta_{k}}\right\} \\
= & P_{\mathrm{t}, S_{k_{x^{*}}}} N_{k} L_{0}\left[\int_{0}^{d} f_{\left\|\mathbf{x}_{S_{k^{*}}}\right\|}(x) d^{-\eta_{k}} d x\right. \\
& \left.+\int_{d}^{\infty} f_{\mathbf{x}_{S_{k_{x^{*}}}} \|}(x) x^{-\eta_{k}} d x\right]
\end{aligned}
$$

In (A.1), the PDF of $\left\|\mathbf{x}_{S_{k^{*}}}\right\|$ is given by [25]

$$
f_{\left\|\mathbf{x}_{B_{k^{*}}}\right\|}(x)=\frac{x}{\Pi_{k}} \exp \left\{-\sum_{j=1}^{K} \zeta_{k, j} x^{2 \eta_{k} / \eta_{j}}\right\},
$$

where $\Pi_{k}$ is given in (11), $\zeta_{k, j}$ and $\rho_{j, k}$ are given in (12) and (13), respectively.

We then turn our attention to the expectation of aggregate interference, which is derived as

$$
\begin{aligned}
\mathbb{E}\left\{I_{S_{x}}\right\}= & \sum_{j=1}^{K} \mathbb{E}_{h}\left\{P_{\mathrm{t}, j} L_{0}\left|\mathbf{h}_{S_{x} u_{0}} \frac{\mathbf{g}_{S_{x} u_{j}}^{H}}{\left\|\mathbf{g}_{S_{x} u_{j}}\right\|}\right|^{2}\right\} \\
& \mathbb{E}_{S_{x^{*}}}\left\{\mathbb{E}_{\Phi_{j}}\left\{\sum_{S_{x} \in \Phi_{j} \backslash \mathcal{S}_{x^{*}}}\left(\max \left\{\left\|\mathbf{x}_{S_{x} u_{0}}\right\|, d\right\}\right)^{-\eta_{j}}\right\}\right\} .
\end{aligned}
$$

Given the distance between the typical MT and the serving BS as $\left\|x_{S_{k^{*}}}\right\|=x$, the interfering BSs need to be located outside a disc of radius $\rho_{j, k}{ }^{1 / \eta_{j}} x^{\eta_{k} / \eta_{j}}$ to satisfy the BRP cell association. Therefore, the radius between the interfering BSs and the typical MT should be larger than $r_{I, \min }=$ $\rho_{j, k}{ }^{1 / \eta_{j}} x^{\eta_{k} / \eta_{j}}$. We proceed by applying the Campbell's theorem to (A.3) to derive

$$
\begin{aligned}
& \mathbb{E}\left\{I_{S_{x}}\right\}=\sum_{j=1}^{K} \mathbb{E}\left\{P_{\mathrm{t}, j} L_{0}\left|\mathbf{h}_{S_{x} u_{0}} \frac{\mathbf{g}_{S_{x} u_{j}}^{H}}{\left\|\mathbf{g}_{S_{x} u_{j}}\right\|}\right|^{2}\right\} \\
& \mathbb{E}_{S_{x^{*}}}\left\{\lambda_{j} \int_{R^{2} / r_{I, \min }^{2}}(\max \{x, d\})^{-\eta_{j}} d x\right\} \\
& =\sum_{j=1}^{K} 2 \pi P_{\mathrm{t}, j} L_{0} \lambda_{j} \int_{0}^{\infty} \int_{r_{I, \min }}^{\infty}(\max \{r, d\})^{-\eta_{j}} r d r \\
& f_{\left\|\mathbf{x}_{S_{k} *}\right\|}(x) d x .
\end{aligned}
$$


By inserting $r_{I, \text { min }}=\rho_{j, k}^{1 / \eta_{j}} x^{\eta_{j} / \eta_{k}}$ into $\mathbb{E}\left\{I_{S_{x}}\right\}$, we have $\begin{aligned} \mathbb{E}\left\{I_{S_{x}}\right\}= & \sum_{j=1}^{K} 2 \pi P_{\mathrm{t}, j} L_{0} \lambda_{j}\left[\int_{0}^{x_{d}}\left(d^{-\eta_{j}} \int_{\rho_{j, k}}^{d / \eta_{j} x^{\eta_{k} / \eta_{j}}} r d r\right.\right. \\ & \left.+\int_{d}^{\infty} r^{-\left(\eta_{j}-1\right)} d r\right) f_{\left\|\mathbf{x}_{S_{k_{x^{*}}}}\right\|}(x) d x+ \\ & \left.\int_{x_{d}}^{\infty} \int_{\rho_{j, k}{ }^{1 / \eta_{j}} x^{\eta_{k} / \eta_{j}}}^{\infty} r^{-\left(\eta_{j}-1\right)} r d r f_{\left\|\mathbf{x}_{S_{k_{x^{*}}}}\right\|}(x) d x\right],\end{aligned}$

where $x_{d}=d^{\eta_{j} / \eta_{k}} \rho_{j, k}{ }^{-1 / \eta_{k}}$.

Substituting the PDF of $\left\|\mathbf{x}_{S_{k_{x^{*}}}}\right\|$ in (A.2) into (A.5), we obtain $\mathbb{E}\left\{I_{S_{x}}\right\}$.

Combining $\mathbb{E}\left\{I_{S_{k_{x}}}\right\}$ in (A.1) and $\mathbb{E}\left\{I_{S_{x}}\right\}$ in (A.5), we derive the maximum transmit power at the typical MT associated with the $k$ th tier as Theorem 1.

\section{APPENDix B}

\section{A Proof of Theorem 2}

According to (4) and (21), the UL outage probability of the typical MT in the $k$ th tier is written as

$$
\begin{aligned}
& P_{\text {out }, k}\left(R_{S}\right)=1-\int_{0}^{\infty} \operatorname{Pr}\left[\frac{\left\|\mathbf{h}_{\mathrm{u}_{0}, S_{k_{x^{*}}}}\right\|^{2}\left\|\mathbf{x}_{\mathrm{u}_{0}, S_{k_{x^{*}}}}\right\|^{-\eta_{k}}}{\left(I_{U}+\sigma^{2}\right) \tau_{k}}>\gamma_{t h}\right] \\
& f_{\left\|\mathbf{x}_{\mathrm{u}_{0}, S_{k_{x^{*}}}}\right\|}(x) d x \text {. }
\end{aligned}
$$

In (B.1), $f_{\|_{\mathbf{x}_{0}, S_{2}}}(x)$ is given in (A.2). The complementary cumulative distribution function (CCDF) of the typical MT at distance $\mathbf{x}$ from its serving BS in $k$ th tier is given by

$\operatorname{Pr}\left[\frac{\left\|\mathbf{h}_{\mathrm{u}_{0}, S_{k_{x^{*}}}}\right\|^{2}\left\|\mathbf{x}_{\mathrm{u}_{0}, S_{k_{x^{*}}}}\right\|^{-\eta_{k}}}{\left(I_{U}+\sigma^{2}\right) \tau_{k}}>\gamma_{t h}\right]$

$=\mathbb{E}_{I_{U}}\left\{\operatorname{Pr}\left[\left\|\mathbf{h}_{\mathrm{u}_{0}, B_{k_{x^{*}}}}\right\|^{2}>\left(I_{U}+\sigma^{2}\right) \gamma_{t h} \tau_{k}\left\|\mathbf{x}_{\mathrm{u}_{0}, B_{k_{x^{*}}}}\right\|^{\eta_{k}}\right] \mid I_{U}\right\}$

$=\sum_{m=0}^{N-1} \frac{1}{m !} \int_{0}^{\infty} \exp \left\{-\left(\tau+\sigma^{2}\right) \gamma_{t h} \tau_{k}\left\|\mathbf{x}_{\mathrm{u}_{0}, B_{k_{x^{*}}}}\right\|^{\eta_{k}}\right\}$

$\left(\left(\tau+\sigma^{2}\right) \gamma_{t h} \tau_{k}\left\|\mathbf{x}_{\mathrm{u}_{0}, B_{k^{*}}}\right\|^{\eta_{k}}\right)^{m} d \operatorname{Pr}\left(I_{U} \leq \tau\right)$.

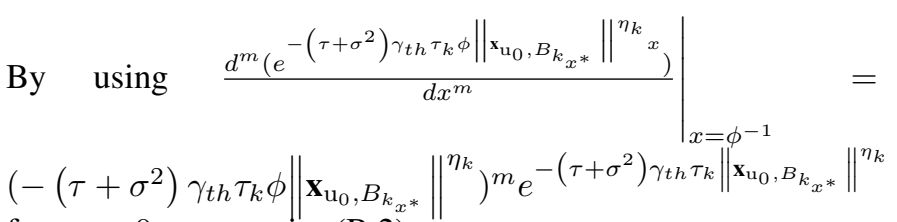
for $m>0$, we rewrite (B.2) as

$$
\begin{aligned}
& \operatorname{Pr}\left[S I N R_{k}>\gamma_{t h}\right]=\exp \left\{-\sigma^{2} \gamma_{t h} \tau_{k}\left\|\mathbf{x}_{\mathrm{u}_{0}, S_{k_{x^{*}}}}\right\|^{\eta_{k}}\right\} \\
& \mathcal{L}_{I_{U}}\left(\gamma_{t h} \tau_{k}\left\|\mathbf{x}_{\mathrm{u}_{0}, S_{k_{x^{*}}}}\right\|^{\eta_{k}}\right)+\sum_{m=1}^{N-1} \frac{\phi^{-m}}{(-1)^{m} m !} \\
& \left.\frac{d^{m}\left(\frac{\mathcal{L}_{I_{U}}\left(\gamma_{t h} \tau_{k} \phi\left\|\mathbf{x}_{\mathbf{u}_{0}, S_{k_{x^{*}}}}\right\|^{\eta_{k}} x\right)}{e^{\sigma^{2} \gamma_{t h} \tau_{k} \phi}\left\|\mathbf{x}_{\mathbf{u}_{0}, S_{k_{x^{*}}}}\right\|^{\eta_{k}}{ }_{x}}\right)}{d x^{m}}\right|_{x=\phi^{-1}} .
\end{aligned}
$$

The Laplace transform of $I_{U}$ is $\mathcal{L}_{I_{U}}(s)=\prod_{j=1}^{K} \mathcal{L}_{I_{U, j}}(s)$. Here, we set $H_{j}=\left.\left|\frac{\mathbf{h}_{\mathrm{u}_{x, j}, B_{x, j}}^{H}}{\| \mathbf{h}_{\mathrm{u}_{x, j}, B_{x, j}}^{H}}\right| \mathbf{h}_{\mathrm{u}_{0}, \mathrm{u}_{x, j}}\right|^{2}$. According to the Generating functional of homogeneous PPP in [40], we derive ${ }^{3}$

$$
\begin{aligned}
& \mathcal{L}_{I_{U, j}}(s)=\exp \left\{-\int\left[1-\mathbb{E}_{H_{j}}\left(\exp \left(-s \tau_{j} H_{j} r^{-\alpha}\right)\right)\right] \lambda_{j} 2 \pi r d r\right\} \\
& \quad=\exp \left\{-\sum_{j=1}^{K} \lambda_{j} \pi \tau_{j}^{\frac{2}{\eta_{j}}} \Gamma\left(1+\frac{2}{\eta_{j}}\right) \Gamma\left(1-\frac{2}{\eta_{j}}\right) s^{\frac{2}{\eta_{j}}}\right\} .
\end{aligned}
$$

Substituting (B.4) into (B.3), we derive

$\operatorname{Pr}\left[S I N R_{k}>\gamma_{t h}\right]=$

$\exp \left\{-\sigma^{2} \gamma_{t h} \tau_{k}\left\|\mathbf{x}_{\mathrm{u}_{0}, S_{k_{x^{*}}}}\right\|^{\eta_{k}}-\sum_{j=1}^{K} \vartheta_{j}\left\|\mathbf{x}_{\mathrm{u}_{0}, S_{k_{x^{*}}}}\right\|^{\frac{2 \eta_{k}}{\eta_{j}}}\left(\gamma_{t h}\right)^{\frac{2}{\eta_{j}}}\right\}$

$+\sum_{m=1}^{N-1} \frac{\phi^{-m}}{(-1)^{m} m !} \frac{d^{m}}{d x^{m}}\left[\exp \left\{-\sigma^{2} \gamma_{t h} \tau_{k} \phi\left\|\mathbf{x}_{\mathrm{u}_{0}, S_{k_{x^{*}}}}\right\|^{\eta_{k}} x\right.\right.$

$\left.\left.-\sum_{j=1}^{K} \vartheta_{k, j}\left\|\mathbf{x}_{\mathrm{u}_{0}, S_{k_{x^{*}}}}\right\|^{\frac{2 \eta_{k}}{\eta_{j}}}\left(\phi \gamma_{t h}\right)^{\frac{2}{\eta_{j}}} x^{\frac{2}{\eta_{j}}}\right\}\right]\left.\right|_{x=\phi^{-1}}$,

where

$$
\begin{aligned}
\vartheta_{k, j}= & \pi \lambda_{j}\left(E\left\{P_{r_{\mathrm{u}_{j}}, j}\right\} / E\left\{P_{\mathrm{r}_{\mathrm{u}_{0}}, k}\right\}\right)^{\frac{2}{\eta_{j}}} \\
& \Gamma\left(1+\frac{2}{\eta_{j}}\right) \Gamma\left(1-\frac{2}{\eta_{j}}\right) .
\end{aligned}
$$

We assume $A(x)=-\sigma^{2} \gamma_{t h} \tau_{k} \phi\left\|\mathbf{x}_{\mathrm{u}_{0}, S_{k_{x^{*}}}}\right\|^{\eta_{k}} x-$ $\sum_{j=1}^{K} \vartheta_{k, j}\left\|\mathbf{x}_{\mathrm{u}_{0}, S_{k_{x^{*}}}}\right\|^{\frac{2 \eta_{k}}{\eta_{j}}}\left(\phi \gamma_{t h}\right)^{\frac{2}{\eta_{j}}} x^{\frac{2}{\eta_{j}}}$, then applying the Faà di Bruno's formula [41], we obtain Theorem 2.

\section{APPENDiX C}

\section{A Proof of Theorem 3}

Based on (36), the UL average ergodic rate of a typical MT associated with the $k$ th tier is derived as

$$
\begin{aligned}
R_{k}= & \int_{0}^{\infty} E_{S I N R_{k}}\left[(1-\alpha) T \ln \left(1+\operatorname{SINR}_{k}(x)\right)\right] \\
& f_{\left\|x_{\mathrm{u}_{0}, B_{k_{x^{*}}}}\right\|}(x) d x \\
= & \int_{0}^{\infty} \int_{0}^{\infty} \operatorname{Pr}\left[\operatorname{SIN} R_{k}(x)>e^{t /((1-\alpha) T)}-1\right] d t \\
& f_{\left\|x_{\mathrm{u}_{0}, B_{k_{x^{*}}}}\right\|}(x) d x .
\end{aligned}
$$

Substituting (4) into (C.1), we obtain Theorem 2.

${ }^{3}$ Note that the average number of active users ( the density of active MTs) is identical to the average number of BSs (BS density) in each tier of this network area. 


\section{APPENDIX D \\ A Proof of Theorem 4}

Based on (49), the UL outage probability of a typical MT associated with the $k$ th tier as $N \rightarrow \infty$ can be given as

$$
\begin{aligned}
& P_{\text {out }, k}^{\infty}\left(R_{S}\right) \\
= & 1-\int_{0}^{\infty} F_{I_{U}}\left(\phi \mathbb{E}\left\{P_{r_{\mathrm{u}_{0}}, k}\right\} N_{k} L_{0}\left\|\mathbf{x}_{\mathrm{u}_{0}, S_{k_{x^{*}}}}\right\|^{-\eta_{k}}\left(\gamma_{t h}\right)^{-1}\right. \\
& \left.-\sigma^{2}\right) f_{\left\|\mathbf{x}_{\mathrm{u}_{0}, S_{k_{x^{*}}}}\right\|}(x) d x .
\end{aligned}
$$

We then utilize the Gil-Pelaez theorem [42] to derive the $\mathrm{CDF}$ of $I_{U}$ as

$$
F_{I_{U}}(x)=\frac{1}{2}-\frac{1}{\pi} \int_{0}^{\infty} \frac{\operatorname{Im}\left[e^{-j w x} \phi^{*}(w)\right]}{w} d w .
$$

Substituting (D.2) into (D.1), we derive the UL outage probability of a typical MT associated with the $k$ th tier as $N \rightarrow \infty$ as (50).

\section{REFERENCES}

[1] Y. Deng, L. Wang, M. Elkashlan, M. D. Renzo, and J. Yuan, "K-tier heterogeneous cellular networks with wireless power transfer," in Proc. IEEE Int. Conf. Commun. (ICC), Jun. 2016.

[2] K. Huang and $X$. Zhou, "Cutting the last wires for mobile communications by microwave power transfer," IEEE Commun. Mag., vol. 53, no. 6, pp. 86-93, Jun. 2015.

[3] S. Bi, C. Ho, and R. Zhang, "Wireless powered communication: opportunities and challenges," IEEE Commun. Mag., vol. 53, no. 4, pp. 117-125, Apr. 2015.

[4] K. Huang, C. Zhong, and G. Zhu, "Some new trends in wirelessly powered communications," CoRR, vol. abs/1508.03728, 2015. [Online]. Available: http://arxiv.org/abs/1508.03728

[5] D. Mishra, S. De, S. Jana, S. Basagni, K. Chowdhury, and W. Heinzelman, "Smart RF energy harvesting communications: challenges and opportunities," IEEE Commun. Mag., vol. 53, no. 4, pp. 70-78, Apr. 2015.

[6] X. Lu, P. Wang, D. Niyato, D. I. Kim, and Z. Han, "Wireless networks with RF energy harvesting: A contemporary survey," IEEE Commun. Surveys and Tutorials, vol. 17, no. 2, pp. 757-789, Secondquarter 2015.

[7] A. H. Sakr and E. Hossain, "Analysis of K-tier uplink cellular networks with ambient RF energy harvesting," IEEE J. Sel. Areas Commun., vol. 33, no. 10, pp. 2226-2238, Oct. 2015.

[8] J. G. Andrews, S. Buzzi, W. Choi, S. V. Hanly, A. Lozano, A. C. K. Soong, and J. C. Zhang, "What will 5G be?" IEEE J. Sel. Areas Commun., vol. 32, no. 6, pp. 1065-1082, Jun. 2014.

[9] R. Madan, J. Borran, A. Sampath, N. Bhushan, A. Khandekar, and T. Ji, "Cell association and interference coordination in heterogeneous LTEA cellular networks," IEEE J. Sel. Areas Commun., vol. 28, no. 9, pp. 1479-1489, Dec. 2010.

[10] A. Ghosh, N. Mangalvedhe, R. Ratasuk, B. Mondal, M. Cudak, E. Visotsky, T. A. Thomas, J. G. Andrews, P. Xia, H. S. Jo, H. S. Dhillon, and T. D. Novlan, "Heterogeneous cellular networks: From theory to practice," IEEE Commun. Mag., vol. 50, no. 6, pp. 54-64, Jun. 2012.

[11] Y. Deng, L. Wang, K.-K. Wong, A. Nallanathan, M. Elkashlan, and S. Lambotharan, "Safeguarding massive mimo aided hetnets using physical layer security," in Int. Wireless Commun. Signal Process. (WCSP), Oct. 2015, pp. 1-5.

[12] K. Huang and V. K. N. Lau, "Enabling wireless power transfer in cellular networks: Architecture, modeling and deployment," IEEE Trans. Wireless Commun., vol. 13, no. 2, pp. 902-912, Feb. 2014.

[13] J. G. Andrews, "Seven ways that hetnets are a cellular paradigm shift," IEEE Commun. Mag., vol. 51, no. 3, pp. 136-144, Mar. 2013.

[14] H. Ju and R. Zhang, "Throughput maximization in wireless powered communication networks," IEEE Trans. Commun., vol. 13, no. 1, pp. 418-428, Jan. 2014.

[15] L. Liu, R. Zhang, and K.-C. Chua, "Multi-antenna wireless powered communication with energy beamforming," IEEE Trans. Commun., vol. 62 , no. 12 , pp. 4349-4361, Dec. 2014.
[16] H. Ju and R. Zhang, "User cooperation in wireless powered communication networks," Proc. IEEE GLOBECOM, pp. 1430-1435, Dec. 2014.

[17] X. Kang, C. K. Ho, and S. Sun, "Full-duplex wireless-powered communication network with energy causality," IEEE Trans. Wireless Commun., vol. 14, no. 10, pp. 5539-5551, Oct. 2015.

[18] G. Yang, C. K. Ho, R. Zhang, and Y. L. Guan, "Throughput optimization for massive MIMO systems powered by wireless energy transfer," IEEE J. Sel. Areas Commun., vol. 33, no. 8, pp. 1640-1650, Aug. 2015.

[19] A. H. Sakr and E. Hossain, "Cognitive and energy harvesting-based D2D communication in cellular networks: Stochastic geometry modeling and analysis," IEEE Trans. Commun., vol. 63, no. 5, pp. 1867-1880, May 2015.

[20] S. Akbar, Y. Deng, A. Nallanathan, M. Elkashlan, and A. H. Aghvami, "Simultaneous wireless information and power transfer in k-tier heterogeneous cellular networks," IEEE Trans. Wireless Commun., no. 99, pp. 1-1, May 2016.

[21] M. Haenggi, Stochastic geometry for wireless networks. Cambridge University Press, 2012.

[22] H. S. Dhillon, R. K. Ganti, F. Baccelli, and J. G. Andrews, "Modeling and analysis of K-tier downlink heterogeneous cellular networks," IEEE J. Sel. Areas Commun., vol. 30, no. 3, pp. 550-560, Apr. 2012.

[23] H. ElSawy and E. Hossain, "On stochastic geometry modeling of cellular uplink transmission with truncated channel inversion power control," IEEE Trans. Wireless Commun., vol. 13, no. 8, pp. 4454-4469, Aug. 2014.

[24] X. Chen and R. Q. Hu, "Joint uplink and downlink optimal mobile association in a wireless heterogeneous network," in Proc. IEEE GLOBECOM, Dec. 2012, pp. 4131-4137.

[25] H.-S. Jo, Y. J. Sang, P. Xia, and J. G. Andrews, "Heterogeneous cellular networks with flexible cell association: A comprehensive downlink SINR analysis," IEEE Trans. Wireless Commun., vol. 11, no. 10, pp. 34843495, Oct. 2012.

[26] J. G. Andrews, F. Baccelli, and R. K. Ganti, "A tractable approach to coverage and rate in cellular networks," IEEE Trans. Wireless Commun., vol. 59, no. 11, pp. 3122-3134, Nov. 2011.

[27] H.-S. Jo, Y. J. Sang, P. Xia, and J. G. Andrews, "Heterogeneous cellular networks with flexible cell association: A comprehensive downlink SINR analysis," IEEE Trans. Wireless Commun., vol. 11, no. 10, pp. 3484 3495, Oct. 2012.

[28] J. Liu, S. Zhang, H. Nishiyama, N. Kato, and J. Guo, "A stochastic geometry analysis of D2D overlaying multi-channel downlink cellular networks," in IEEE INFOCOM, Apr. 2015, pp. 46-54.

[29] H. ElSawy, E. Hossain, and M.-S. Alouini, "Analytical modeling of mode selection and power control for underlay D2D communication in cellular networks," IEEE Trans. Commun., vol. 62, no. 11, pp. 41474161, Nov. 2014

[30] T. D. Novlan, H. S. Dhillon, and J. G. Andrews, "Analytical modeling of uplink cellular networks," IEEE Trans. Wireless Commun., vol. 12 , no. 6, pp. 2669-2679, Jun. 2013.

[31] F. Baccelli and B. Blaszczyszyn, Stochastic geometry and wireless networks: Volume 1: Theory. Now Publishers Inc, 2009, vol. 1.

[32] F. Baccelli, B. Blaszczyszyn, and P. Muhlethaler, "An aloha protocol for multihop mobile wireless networks," IEEE Trans. Inf. Theory, vol. 52, no. 2, pp. 421-436, Feb. 2006.

[33] R. W. Heath, M. Kountouris, and T. Bai, "Modeling heterogeneous network interference using poisson point processes," IEEE Trans. Signal Process., vol. 61, no. 16, pp. 4114-4126, Aug. 2013.

[34] E. U. T. R. Access, "Further advancements for E-UTRA physical layer aspects," 3GPP TR 36.814, Tech. Rep., 2010.

[35] H. Q. Ngo, M. Matthaiou, T. Q. Duong, and E. G. Larsson, "Uplink performance analysis of multicell MU-MIMO systems with ZF receivers," IEEE Trans. Veh. Technol., vol. 62, no. 9, pp. 4471-4482, Nov. 2013.

[36] K. Smiljkovikj, P. Popovski, and L. Gavrilovska, "Analysis of the decoupled access for downlink and uplink in wireless heterogeneous networks," [Online]. Available: arxiv.org/abs/1407.0536, 2014.

[37] K. Huang, "Spatial throughput of mobile ad hoc networks powered by energy harvesting," IEEE Trans. Inf. Theory, vol. 59, no. 11, pp. 75977612, Nov. 2013.

[38] X. Zhou, R. Zhang, and C. K. Ho, "Wireless information and power transfer: architecture design and rate-energy tradeoff," in Proc. IEEE GLOBECOM, 2012, pp. 3982-3987.

[39] I. S. Gradshteyn and I. M. Ryzhik, Table of Integrals, Series and Products, 7th ed. San Diego, C.A.: Academic Press, 2007.

[40] D. Stoyan, W. Kendall, and J. Mecke, "Stochastic geometry and its applications," Wiley New York, vol. 2, 1987.

[41] S. Roman, "The formula of faa di bruno," American Mathematical Monthly, pp. 805-809, 1980. 
[42] J. G. Wendel, "The non-absolute convergence of Gil-Pelaez' inversion integral," The Annals of Mathematical Statistics, vol. 32, no. 1, pp. 338339, Mar. 1961. 